\title{
Intraperitoneal chemotherapy for ovarian cancer with peritoneal metastases, systematic review of the literature and focused personal experience
}

\author{
Federico Coccolini ${ }^{1}$, Paola Fugazzola ${ }^{2}$, Giulia Montori ${ }^{3}$, Luca Ansaloni ${ }^{2}$, Massimo Chiarugi $^{1}$ \\ ${ }^{1}$ General, Emergency and Trauma Surgery, Pisa University Hospital, Pisa, Italy; ${ }^{2}$ General, Emergency and Trauma Surgery, Bufalini Hospital, \\ Cesena, Italy; ${ }^{3}$ General Surgery, Aviano Hospital, Aviano, Italy \\ Contributions: (I) Conception and design: F Coccolini; (II) Administrative support: None; (III) Provision of study materials or patients: None; (IV) \\ Collection and assembly of data: None; (V) Data analysis and interpretation: None; (VI) Manuscript writing: All authors; (VII) Final approval of \\ manuscript: All authors. \\ Correspondence to: Federico Coccolini, MD. General, Emergency and Trauma Surgery, Pisa University Hospital, Via Paradisa, 2, 56124 Pisa, Italy. \\ Email: federico.coccolini@gmail.com.
}

\begin{abstract}
Epithelial ovarian cancer (EOC) causes 60\% of ovarian cancer cases and is the fourth most common cause of death from cancer in women. The standard of care for EOC includes a combination of surgery followed by intravenous chemotherapy. Intraperitoneal (IP) chemotherapy (CT) has been introduced into the therapeutic algorithm of EOC with positive results. To explore existing results regarding intraperitoneal chemotherapy a systematic review of the literature and an analysis of our own institutional prospective database of patients treated with cytoreductive surgery plus hyperthermic intraperitoneal chemotherapy (HIPEC) for EOC at different stages were conducted. The focused report concerning our personal experience with advanced EOC treated with cytoreductive surgery and HIPEC produced the following results: In 57 patients cisplatin + paclitaxel as HIPEC was the only significant factor improving overall survival (OS) at multivariate analysis (OR 6.54, 95\% CI: 1.24-34.47, P=0.027). Patients treated with HIPEC cisplatin + paclitaxel showed a median OS of 46 months (SD 6.4, 95\% CI: 33.4-58.6), while patients treated with other HIPEC regimens showed a median OS of 12 months (SD 3.1, 95\% CI: 6.0-18.0). The $2 \mathrm{y}$-OS was $72 \%$ and $3 \mathrm{y}$-OS was $68 \%$ for cisplatin + paclitaxel as HIPEC, while the $2 \mathrm{y}$ - and $3 \mathrm{y}$-OS was $0 \%$ for other HIPEC regimens. Patients treated with HIPEC cisplatin + paclitaxel showed a median disease-free survival (DFS) of 13 months (SD 1.6, 95\% CI: 9.9-16.1), while patients treated with other HIPEC regimens showed a median DFS of 8 months (SD 3.1, 95\% CI: 1.9-14.1). In conclusion, HIPEC cisplatin + paclitaxel in ovarian cancer showed positive results that may be considered semi-definitive according to the level of evidence and should be considered a starting point for further investigations. At present HIPEC cisplatin + paclitaxel should be proposed to patients with advanced ovarian cancer as standard treatment at almost all stages of disease. Platinum + taxane-based intraperitoneal regimens demonstrated superior results compared to other regimens.
\end{abstract}

Keywords: Ovarian; cancer; chemotherapy; intraperitoneal chemotherapy; upfront surgery; consolidation surgery; surgery for recurrence; salvage surgery; cisplatin; paclitaxel

Submitted Jul 16, 2020. Accepted for publication Oct 13, 2020.

doi: 10.21037/jgo-2020-06

View this article at: http://dx.doi.org/10.21037/jgo-2020-06

@ Journal of Gastrointestinal Oncology. All rights reserved. 


\section{Introduction}

Epithelial ovarian cancer (EOC) causes $60 \%$ of ovarian cancer cases and is the fourth most common cause of death from cancer in women. The most frequent histologic type ( $70 \%$ of cases) is high-grade serous ovarian cancer with a typical biological behavior. According to FIGO classification the stage III includes a tumor with involvement of one or both ovaries and/or the Fallopian tubes with peritoneal involvement, outside the pelvis (FIGO IIIb) and retroperitoneal lymph node involvement (FIGO IIIc) (1). Stages IIIb and IIIc comprise about $60 \%$ of EOC. The standard of care for EOC includes a surgical removal of all visible evidence of disease by extensive cytoreductive surgery (CRS). This includes hysterectomy and bilateral salpingo-oophorectomy, total omentectomy, appendectomy (in mucinous histologic types), removal of bulky pelvic and aortic lymph nodes, and removal of all macroscopic disease. The cancer resection is followed by intravenous (IV) chemotherapy (CT), including a platinum-based drug with or without a taxane $(2,3)$.

Recurrence is a common event in high grade EOC, with $75 \%$ of women experiencing relapse within 2 years from diagnosis and subsequent treatment (4). Among patients with recurrent disease, two-third have peritoneal metastases (5). The most investigated factors predicting outcome after recurrence is the platinum-free interval following primary platinum-based chemotherapy and the presence of BRCA mutations (6). Traditionally, most patients with recurrentEOC (rEOC) are treated with chemotherapy alone, the type of which is guided by the platinum sensitivity. Patients with recurrence more than six months after a complete response are considered "platinum-sensitive" (platinum-S) and can be re-treated with platinum-based CT. Patients with persistent disease after front-line treatment or patients who recur within 6 months are considered "platinum-resistant" (platinum-R) and are unlikely to respond to further platinum. In recent decades several studies concerning the role of intraperitoneal antibodies, immunotherapy, radiotherapy and the administration of chemotherapeutic agents directly into the peritoneal cavity before, during or after surgery, have been performed to evaluate their impact on survival.

Intraperitoneal (IP) CT has been introduced into the therapeutic algorithm of EOC with positive but not definitive results. Several methodologies for delivering IP CT have been described. The most common way to perform intraperitoneal chemotherapy is the hyperthermic intraperitoneal chemotherapy (HIPEC). Early postoperative intraperitoneal chemotherapy (EPIC) and the pressurized intraperitoneal aerosolized chemotherapy (PIPAC) have also been described and utilized with interesting results.

EOC HIPEC may be used with variable timing: Primary CRS, secondary CRS, interval debulking, CRS for progressive ovarian cancer, CRS in recurrent EOC and palliative surgery (2).

Our systematic review aims to present the different results of IP CT at different timepoints of the disease and to review the drugs administered intraperitoneally. Moreover, a personal experience describing new results obtained with combined administration of platinum and taxanes as HIPEC will be presented. We present the following article in accordance with the PRISMA 2009 Checklist (available at http://dx.doi.org/10.21037/jgo-2020-06).

\section{Material and methods}

\section{Systematic review}

A computerized search was performed in selected databanks (MEDLINE, Scopus, EMBASE). Citations were included for the period between January 1990 and January 2020 using the primary search strategy: ovarian cancer, intraperitoneal chemotherapy, HIPEC, EPIC, PIPAC, IP, drugs, pharmacokinetic, pharmacodynamic, hyperthermia, outcome, follow-up, consolidative, combined with and/or. No search restrictions were imposed. The dates were selected to allow comprehensive published abstracts of clinical trials, consensus conferences, comparative studies, congresses, guidelines, government publications, multicenter studies, systematic reviews, metaanalysis, large case series, original articles, and randomized controlled trials. Only EOC (serous, mucinous, clear cell, carcinosarcoma, endometrioid, cystadenocarcinoma, adenocarcinoma, Fallopian tube carcinoma, and primary peritoneal malignancies) were included in the study. The research strategy is summarized in Figure 1. Two reviewers (FC and PF) analyzed the literature and selected studies. Were uncertainty arises a thirs reviewer was asked to express his opinion (LA).

\section{Personal experience:}

From our electronic database we selected patients with EOC treated with CRS combined with HIPEC at different 

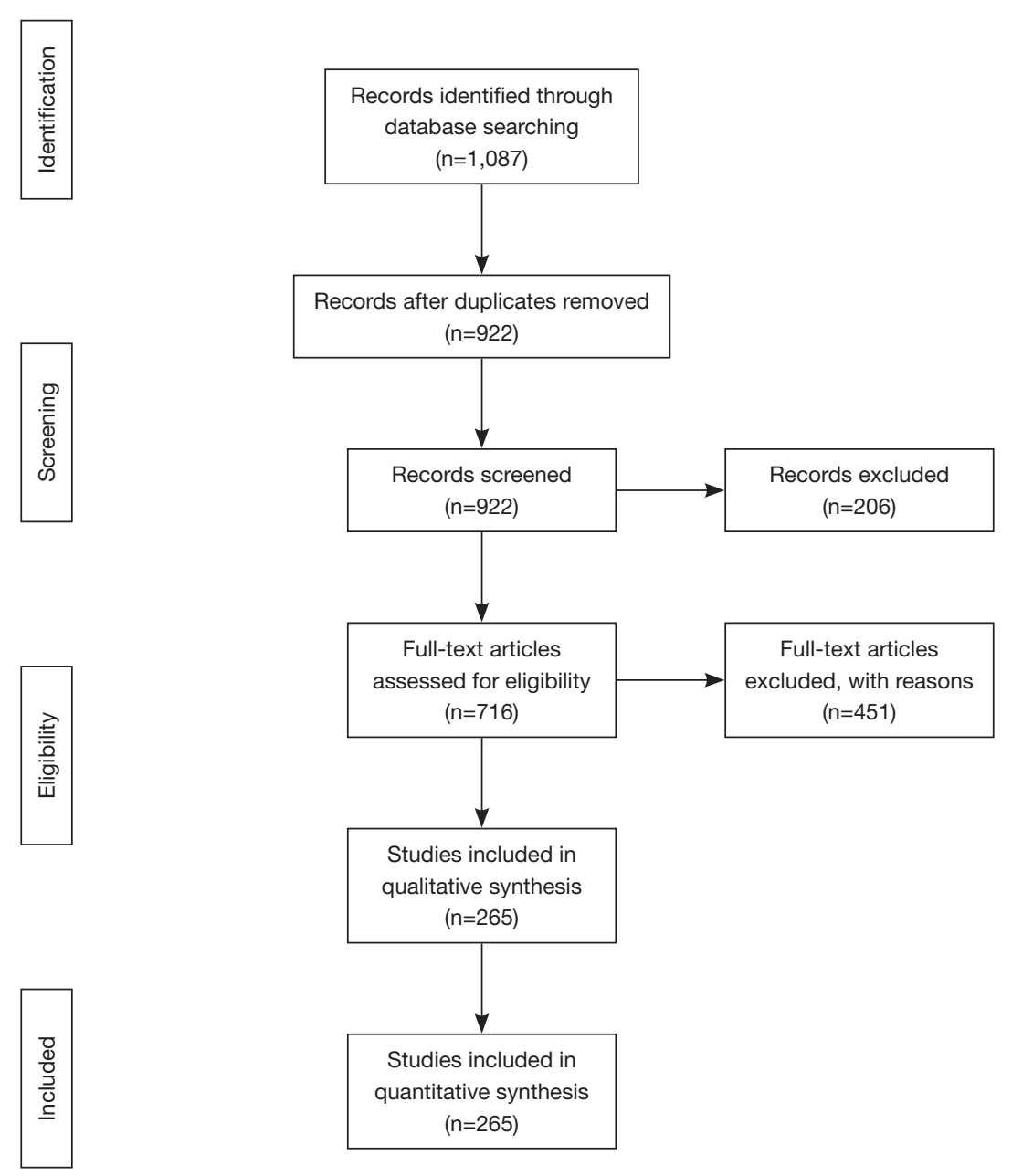

Figure 1 PRISMA flow chart.

time points of the disease (upfront CRS and HIPEC, interval CRS and HIPEC, CRS and HIPEC for recurrent EOC) from January 2011 to May 2019. A retrospective analysis of prospectively collected data was performed. All patients had an Eastern Cooperative Oncology Group (ECOG) performance status $\leq 2$, stage IIIC and IV EOC with resectable disease, no extra-abdominal disease and no significant comorbidities which would preclude the combined treatment. Patients with histology other than EOC and without complete data concerning follow-up were excluded.

The extent of the disease after laparotomy was determined by PCI. The abdomen and pelvis were divided into 13 regions and the size of the lesion was scored as $0-3$. The maximum score was 39. CRS was performed removing all peritoneum and visceral organs involved by the tumor. Omentectomy, appendectomy and cholecystectomy were routinely performed. The completeness of cytoreduction score (CC) was estimated by the surgeon at the conclusion of the procedure according to the following classification: $\mathrm{CC} 0$ - complete cytoreduction of all visible disease; $\mathrm{CC} 1-$ minimal residual disease with nodules less than $2.5 \mathrm{~cm}$; CC2 - residual disease with nodules of $2.5 \mathrm{~mm}$ to $2.5 \mathrm{~cm}$; and CC3 - residual disease with nodules greater than $2.5 \mathrm{~cm}$. 
HIPEC was performed with the "coliseum technique": one inflow and four outflow catheters were placed with the open abdomen that was partially closed with a surgical adhesive drape performing a "closed-HIPEC with open abdomen technique", with a IP temperature was $42-43^{\circ} \mathrm{C}$. HIPEC regimens were: Cisplatin $100 \mathrm{mg} / \mathrm{m}^{2}+$ paclitaxel $175 \mathrm{mg} / \mathrm{m}^{2}$ or cisplatin $100 \mathrm{mg} / \mathrm{m}^{2}+$ mitomycin C $16 \mathrm{mg} / \mathrm{m}^{2}$ or cisplatin $100 \mathrm{mg} / \mathrm{m}^{2}+$ doxorubicin $15.2 \mathrm{mg} / \mathrm{L}$ of perfusate or cisplatin $100 \mathrm{mg} / \mathrm{m}^{2}$ alone. After HIPEC, the perfusate was drained and the reconstruction was performed.

The primary endpoints of the analysis were DFS and OS. Univariate and multivariate analysis were performed to define factors affecting OS and DFS, as secondary endpoint.

\section{Statistical analysis}

DFS and OS were calculated as the interval between the date of CRS and HIPEC and the data of the last followup or of the death or of the recurrence of disease. DFS and OS were calculated with Kaplan-Meier method, and survival estimates were compared using the log-rank test. Multivariate analysis was performed for OS and DFS with Cox regression. Statistical significance was defined as a $P$ value $<0.005$. All analysis was performed using SPSS 20 (IBM Corp, Released 2011, IBM SPSS Statistics for Windows, Version 20.0, Armonk, NY, USA)

\section{Systematic review results}

The focus of the majority of the studies concerning advanced ovarian cancer are tumor biology and behavior of the tumor. Noteworthy, women with mutations of BRCA1 and BRCA2 genes have a higher risk (11-40\%) to develop EOC. Cytoreductive surgery (CRS) has been showed as one of the most important factors influencing survival rates. The aim of CRS is to remove all visible disease, giving a demonstrated survival benefit with increasing completeness of cytoreduction. Intraperitoneal chemotherapy aims to remove residual microscopic disease with an additional positive effect of reducing systemic toxicity as compared to the intravenous CT.

In 2002, a meta-analysis by Bristow et al. analyzed 6,885 women with stage III and IV EOC. They demonstrated that if CRS removed less than $25 \%$ of the disease patients experienced a mean weighted median survival of 22.7 months. IF CRS removed more than $75 \%$ of the disease the mean weighted median survival was 33.9 months. Each $10 \%$ increase in cytoreduction rate was associated with an increase of $5.5 \%$ in median survival time (7). However, at primary surgery, in $74 \%$ and $73 \%$ respectively of women with stage III and stage IV of disease there was lymph node positivity. For this reason, IV CT remains fundamental to reduce or limit lymphatic tumor dissemination and to downstage and downsize the tumor $(2,8)$. Despite aggressive treatment more than $60 \%$ of women had recurrence within $12-18$ months. In general, recurrence is seen in $29.4 \%$ in abdominal cavity and $25.9 \%$ in the pelvis, $7.1 \%$ in retroperitoneal lymph node and $6.3 \%$ in superficial lymph nodes (9). The addition of HIPEC has many goals: To treat microscopical disease, to increase drug penetration into the tissues, to have an intrinsic antitumor effect and to increase the cytotoxicity of some CT drugs. In the open technique it can be manual and uniformly distributed $(3,10)$.

In 2006 a National Cancer Institute (NCI) clinical announcement about EOC (FIGO III-IV) reported that adding IP CT to IV CT significantly improves survival by 12 months (range $0-16$ months) if associated with optimal CRS (CC0-1). However in a recent report, Vergote et al. (8) suggested that IP CT was not a standard of care in first-line treatment for advanced EOC because of the results of the GOG 252 study.

Some reports comparing IP/IV to only IV therapy, showed a possible increasing in toxicity in IP/IV regimens; however, it is short-term and manageable (3). Certain CT agents, including cisplatin and paclitaxel, were found to have distinct pharmacokinetic advantages when administered intraperitoneally (11-13). The American Society of Peritoneal Surface Malignancies (ASPSM) suggests the use of mitomycin-C or paclitaxel especially in platinumresistant disease (3).

\section{Primary epithelial ovarian cancer}

Few trials were published about HIPEC in primary EOC (pEOC). HIPEC for pEOC can be proposed in an upfront setting (U-HIPEC) or as an interval treatment (I-HIPEC) (14). U-HIPEC for primary CRS would be followed by platinum-based adjuvant chemotherapy. However, frequently women with pEOC cannot tolerate primary CRS due to a lack of fitness for major surgery or the extent of disease. In these cases a neoadjuvant chemotherapy followed by CRS plus I-HIPEC may be appropriate.

Three randomized controlled trials (RCT) have been conducted to evaluate HIPEC in upfront or interval setting in pEOC (Table 1). Van Driel in 2018 published the results 

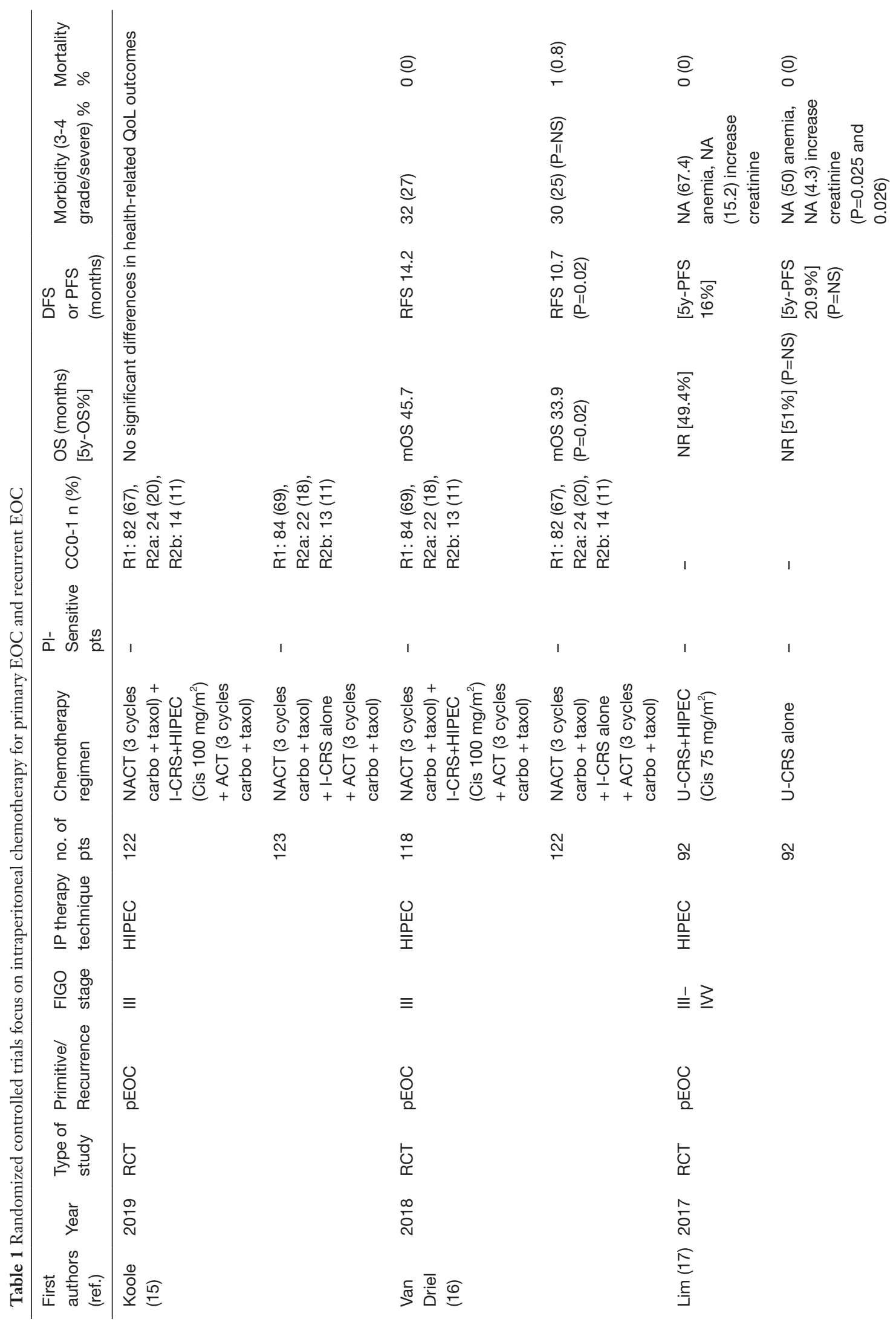

\section{$\frac{\infty}{0} \widehat{\overline{0}} \frac{\mathscr{c}}{\mathrm{O}} \widehat{\overline{0}}$}

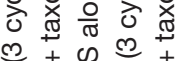

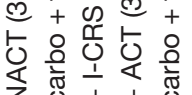

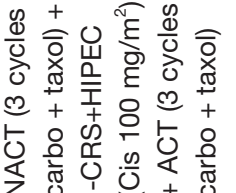

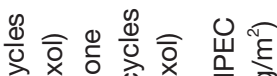

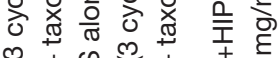

m+

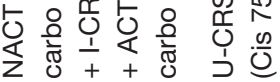

$\stackrel{\Re}{\simeq}$

$\stackrel{\infty}{\mp}$

$\stackrel{\text { กิ }}{2}$

๙

ชิ

㟧

岀

$\equiv$

$\stackrel{1}{\equiv}$

ญ्ठ

음

ঢ

$\underset{\Upsilon}{\mathfrak{X}}$

$\stackrel{\infty}{\stackrel{N}{N}}$

急

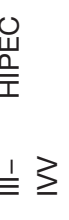

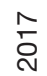

톨 


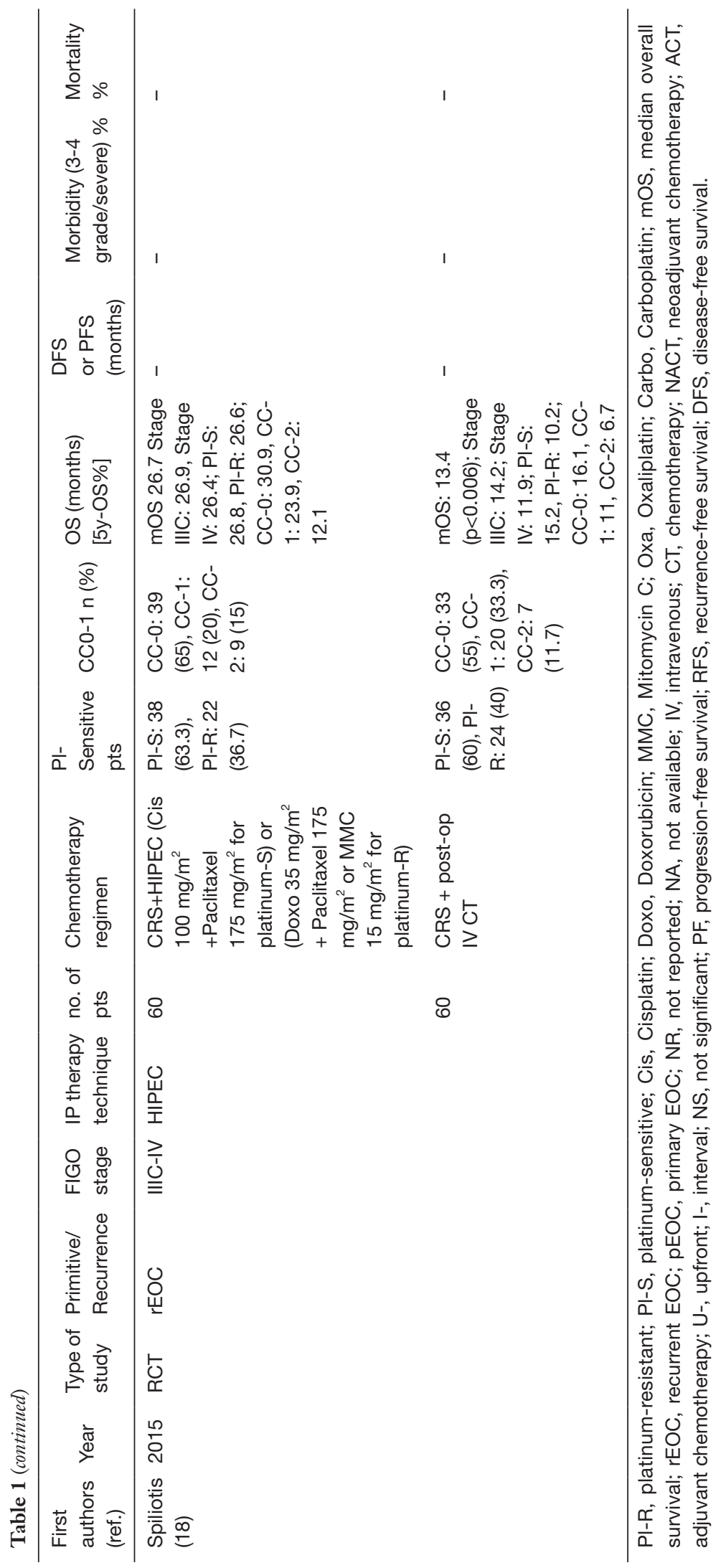

(C) Journal of Gastrointestinal Oncology. All rights reserved. F Gastrointest Oncol 2021;12(Suppl 1):S144-S181 I http://dx.doi.org/10.21037/jgo-2020-06 


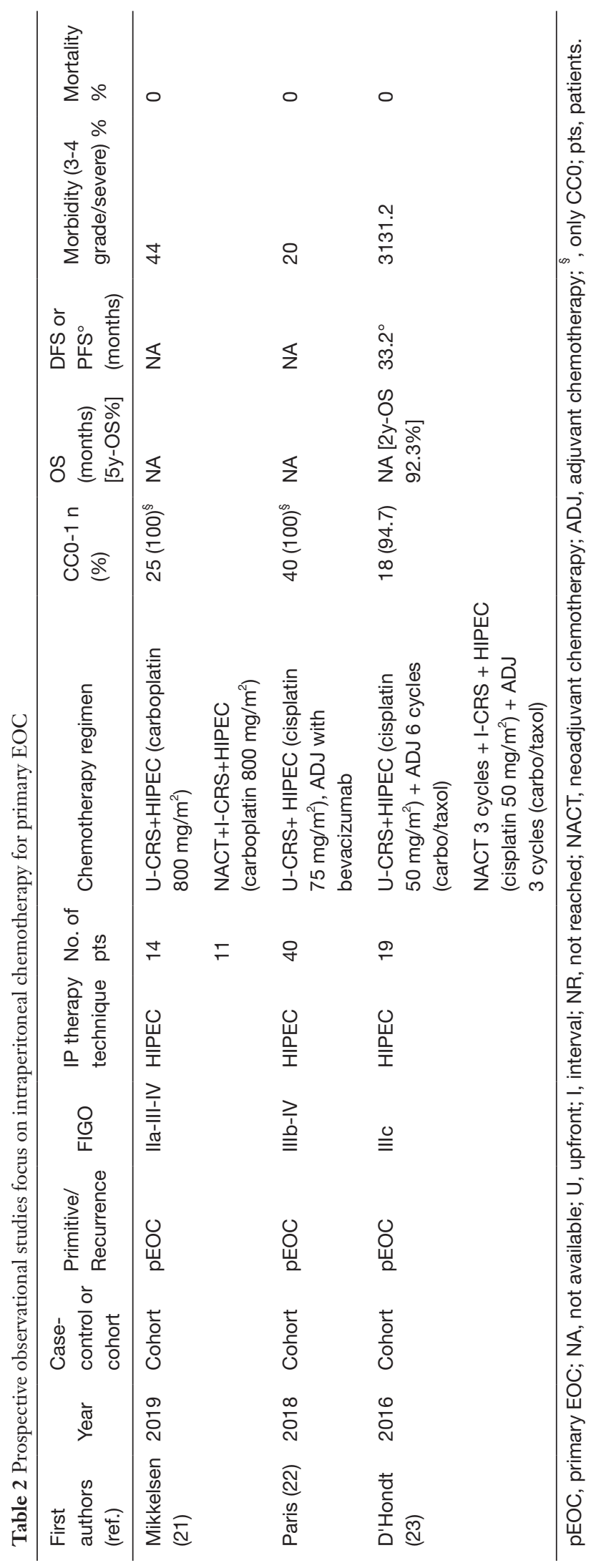

of the OVHIPEC trial (16). 245 patients were randomized to HIPEC and CRS or CRS alone in an interval setting. In the study group a 12 months increase in overall survival (OS) and 4 months increase in progression-free survival (PFS) were demonstrated. Moreover, morbidity and quality of life were similar in the two groups. Survival in the control group (33.9 months) was very similar to the results reported by Chiva $e t a l$. in their meta-analysis concerning primary or interval CRS alone in EOC (33 months). As a comparison I-HIPEC increased median OS to 45.7 months (versus 33.9) without increasing toxicity rate and with a similar quality of life $(15,19)$.

Lim et al., in their RCT in 2017, reported the randomization of 184 patients with similar result both in OS, PFS and mortality in an upfront setting. They reported an increased anemia and acute kidney injury rate in the HIPEC group. However, these authors found in neo-adjuvant chemotherapy (NACT) subgroup an improved outcome in favour of HIPEC and suggested that a longer follow-up may help in showing the real effect of HIPEC (20).

The last study from Koole et al. reported in 2019 results derived from the randomization of 246 patients managed in an interval setting. They did not find significant differences in survival, recurrence or quality of life results (15).

Observational studies reported in literature considered both primary EOC (pEOC) and recurrent EOC (rEOC).

There are three prospective studies analyzing pEOC treated with CRS + HIPEC in an upfront setting (21-23) (Table 2). In more than $94 \%$ of patients a complete cytoreduction (CC0) was achieved. No mortality was reported, and major morbidity ranged between $20 \%$ to $44 \%$. Despite the different drug regimens (Paris et al. added adjuvant bevacizumab) the 2-year OS was 93.2\% (23).

The HYPERO study (20) reported in U-HIPEC setting a mean OS of 41.7 months, with a $2 \mathrm{y}$-OS of $57 \%$ and a $5 \mathrm{y}-\mathrm{OS}$ of $33.3 \%$, and in I-HIPEC setting a mean OS of 68.6 months, with a $2 \mathrm{y}-\mathrm{OS}$ of $80.4 \%$ and a $5 \mathrm{y}-\mathrm{OS}$ of $50.2 \%$.

There are four retrospective studies focused on pEOC. In these studies different HIPEC timings and CT regimens were compared (up-front, interval, or associate with dose-dense chemotherapy) (24-27) (Table 3). CC0 was achieved in more than $73 \%$ of patients. These studies reported a mortality rate lower than $3 \%$ and a morbidity rate of $13-26.5 \%$. The reported DFS ranged between 10 and 35 months. 5-years OS ranged between 31.5 and $46.8 \%$. Biacchi et al. (25), analyzed women with primary advanced tubo-ovarian high-grade serous cancer. They 


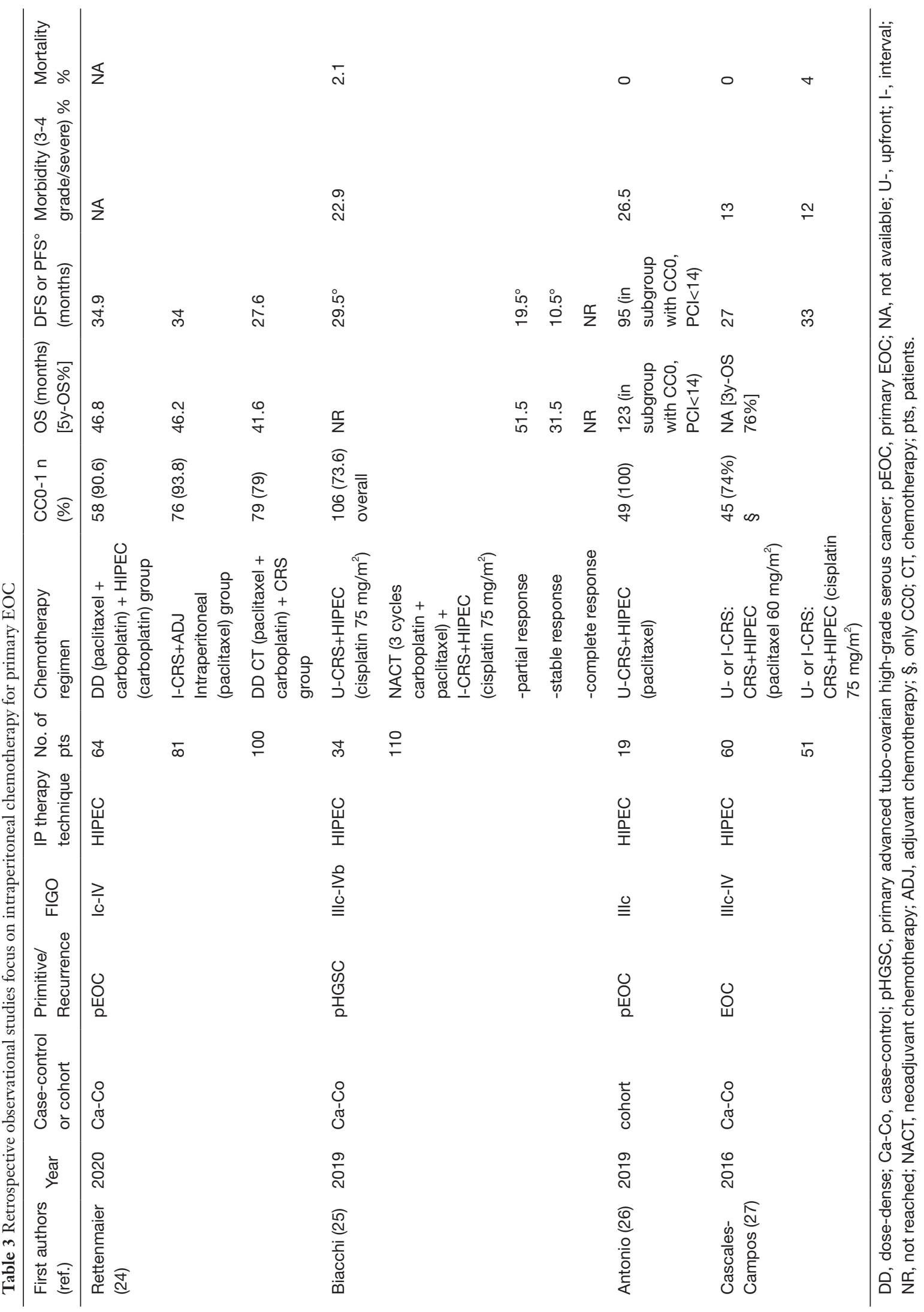

(C) Journal of Gastrointestinal Oncology. All rights reserved. F Gastrointest Oncol 2021;12(Suppl 1):S144-S181 I http://dx.doi.org/10.21037/jgo-2020-06 
showed no difference between U- or I-HIPEC in terms of DFS and OS. U-HIPEC showed similar outcome to patients who underwent I-HIPEC with complete response after NACT. However, the small number of patients undergoing U-HIPEC and the retrospective design limited the reliability of this study. No differences in terms of complications, were reported comparing HIPEC (I- or U-) with cisplatin $\left(75 \mathrm{mg} / \mathrm{m}^{2}\right)$ or paclitaxel $\left(60 \mathrm{mg} / \mathrm{m}^{2}\right)(26)$. Rettenmaier et al. (24) suggested that dose density chemotherapy with HIPEC may offer better results in terms of OS and DFS, especially in BRCA mutated patients. In conclusion, as suggested by van Driel, the optimal timepoint may be the I-HIPEC. The NACT provides a higher rate of $\mathrm{CC} 0$ cytoreduction and can be advantageously associated with HIPEC. Moreover, HIPEC may have a role in reducing peritoneal recurrence in EOC, which has a greater impact on survival than lymph nodal recurrence (28). The role of bevacizumab in front-line setting combined with HIPEC remains to be explored. Lastly, more attention should be paid to the genotypes in evaluating results and approaches. Futher answers might come from from the several ongoing trials (Table 4).

\section{Recurrent epithelial ovarian cancer (rEOC)}

The prognosis of rEOC treated with standard chemotherapy is poor, with a reported median survival of $12-24$ months (29). In a few studies focusing on platinum-S patients, the median OS reaches 35 months, while in platinum-R patients it is about 12 months (22). The need for alternative treatment modalities has been pointed out by Stathopoulos et al., who stated that multiple chemotherapy lines do not offer a survival benefit as compared to one or two lines $(2,30)$.

In recent years, patients with $\mathrm{rEOC}$ with a BRCA mutation (BRCAmut) are most likely to benefit from treatment with PARP inhibitors, after response to a platinum-based chemotherapy, with reported DFS of 11.2 months (compared to 4.3 months of the placebo group) and OS of 34.9 months (31). For patients with wild-type BRCA (BRCAwt) treated with Olaparib, the reported DFS was 7.4 (compared to 5.5 months in the placebo group) and the median OS of 24.5 months.

The role of CRS in rEOC and its role in relation with the patients' BRCA status has recently been clarified by the AGO DESKTOP III/ENGOT ov20 trial results (32). The study showed a significant survival advantage of 7.2 months in platinum-S women with positive AGO score who underwent complete resection. They showed a DFS of 14 months without and of 19.6 months with CRS. BRCAmut patients had the best DFS regardless of having received secondary CRS or not, with a 5 -year DFS of $73 \%$ in non-resected women versus $78 \%$ in resected women $(\mathrm{P}=0.558)$. Conversely, BRCAwt patients who underwent complete CRS had a significantly longer DFS compared with BRCAwt patients who did not receive surgery (5-year DFS of $54 \%$ vs. $42 \%$; $\mathrm{P}=0.048$ ).

\section{HIPEC with recurrent epithelial ovarian cancer}

Many retrospective and prospective observational studies (Tables 5-8) focused on the effects of HIPEC on patients with $\mathrm{rEOC}$ with heterogenous results. In these studies taken together, the reported median OS for rEOC treated with CRS and HIPEC ranges from 24.3 to 58.3 months (5y-OS: 8-79\%) and the median DFS from 6 to 28 months (5y-DFS: 7-30\%). In the largest study by Bakrin et al. (75), on 477 rEOC treated with CRS+HIPEC, the median OS was 45.7 months, with a OS of CC- 0 patients of 52 months (compared to 33 months in not completely cytoreduced patients), without difference between platinum-S and platinum-R patients. In the study by Classe et al. (41) on 314 patients the $5 \mathrm{y}$-OS was $38.0 \%$ (median OS 42 months for platinum-S and 51 months for platinum-R, $\mathrm{P}=0.38$ ) and $5 \mathrm{y}$-DFS was $14 \%$ (median DFS 13 months for platinum-S and 14 months for platinum-R, $\mathrm{P}=0.013$ ). In the study by Bakrin et al. (36) on 246 patients the median OS was 48.9 months (48 months in platinum-R and 52 months in platinum-S) and the $5 \mathrm{y}-\mathrm{OS} 35 \%$; the median DFS was 12.8 months and the 5 y-DFS $9 \%$. Focusing on platinum-S patients treated with CRS+HIPEC, the reported OS ranged from 26 to 58.8 months (5y-OS: $50-79 \%$ ) and the DFS from 6 to 27 (5y-DFS: $30 \%$ ). The reported OS for platinum-R patients ranges from 9 to 51 months (33). Bakrin et al. (36) and Chatzigeorgiou et al. (39) compared OS of platinum-R and platinum-S patients with rEOC treated with CRS + HIPEC without showing significant difference.

Three case-control studies $(33,56,59)$, all focusing on platinum-S patients, compared patients with rEOC treated with CRS+HIPEC with patients treated with traditional systemic CT. In the study by Safra et al. (59) and by Marocco et al. (56) patients treated with CRS and HIPEC showed significantly longer OS and DFS. In the study by Amira et al. (33) there was no significant difference in outcomes of the two groups. Safra et al. (59) compared BRCAmut and BRCAwt patients with rEOC treated with 
Table 4 Ongoing randomized controlled trials about CRS and HIPEC for primary and recurrent EOC found in clinicalregister.gov at March 2020

\begin{tabular}{|c|c|c|c|c|c|c|}
\hline $\begin{array}{l}\text { Clinical trial } \\
\text { number }\end{array}$ & Title of trial & $\begin{array}{l}\text { Type of } \\
\text { tumor }\end{array}$ & Trial design & Country & $\begin{array}{l}\text { Primary/ } \\
\text { Recurrence }\end{array}$ & $\begin{array}{l}\text { State of } \\
\text { trial }\end{array}$ \\
\hline NCT02681432 & $\begin{array}{l}\text { Hyperthermic } \\
\text { Intraperitoneal } \\
\text { Chemotherapy } \\
\text { with Paclitaxel in } \\
\text { Advanced Ovarian } \\
\text { Cancer (HIPECOVA) }\end{array}$ & EOC & $\begin{array}{l}\text { HIPEC-arm: CRS+HIPEC with paclitaxel } \\
\left(175 \mathrm{mg} / \mathrm{m}^{2}\right) \text { for } 60 \text { minutes at a } 42-43^{\circ} \\
\text { degrees; followed by adjuvant systemic IV } \\
\text { chemotherapy with carboplatin (AUC=6) and } \\
\text { paclitaxel }\left(175 \mathrm{mg} / \mathrm{m}^{2}\right) \text { for } 6 \text { cycles } \\
\text { Control arm: CRS followed by adjuvant } \\
\text { systemic IV chemotherapy with carboplatin } \\
(\text { AUC }=6) \text { and paclitaxel }\left(175 \mathrm{mg} / \mathrm{m}^{2}\right) \text { for } \\
6 \text { cycles }\end{array}$ & Spain & $\mathrm{P}$ & Recruiting \\
\hline NCT03842982 & $\begin{array}{l}\text { Hyperthermic } \\
\text { Intraperitoneal } \\
\text { Chemotherapy } \\
\text { (HIPEC) in Ovarian } \\
\text { Cancer (CHIPPI) }\end{array}$ & EOC & $\begin{array}{l}\text { Experimental: Primary Debulking Surgery } \\
\text { (PDS) or Interval Debulking Surgery (IDS) + } \\
\text { Neo or Adjuvant chemotherapy (standard } \\
\text { care) + HIPEC } \\
\text { Control arm: Surgery (Primary Debulking } \\
\text { Surgery (PDS) or Interval Debulking Surgery } \\
\text { (IDS)) + Neo or Adjuvant chemotherapy } \\
\text { ONLY (standard care, without HIPEC) }\end{array}$ & France & $\mathrm{P}$ & Recruiting \\
\hline NCT03373058 & $\begin{array}{l}\text { Efficacy of HIPEC } \\
\text { in the Treatment of } \\
\text { Advanced-Stage } \\
\text { Epithelial Ovarian } \\
\text { Cancer After } \\
\text { Cytoreductive Surgery } \\
\text { (EHTASEOCCS) }\end{array}$ & $\begin{array}{l}\text { EOC, FTC, } \\
\text { PPC }\end{array}$ & $\begin{array}{l}\text { Experimental arm: CRS+HIPEC with } \\
\text { Docetaxel } 75 \mathrm{mg} / \mathrm{m}^{2} \text { and cisplatin } 75 \mathrm{mg} / \mathrm{m}^{2} \\
\text { intraperitoneally in succession, followed by } 6 \\
\text { cycles of adjuvant chemotherapy: paclitaxel } \\
175 \mathrm{mg} / \mathrm{m}^{2} \mathrm{IV}>3 \text { hour (Docetaxel } 75 \mathrm{mg} / \mathrm{m}^{2} \text {, } \\
\text { if paclitaxel is not available) + carboplatin } \\
\text { AUC = 5-6 IV }>1 \text { hour, every } 3 \text { weeks } \\
\text { Control arm: CRS followed by } 6 \text { cycles of } \\
\text { adjuvant chemotherapy: paclitaxel } 175 \\
\mathrm{mg} / \mathrm{m}^{2} \mathrm{IV}>3 \text { hour (Docetaxel } 75 \mathrm{mg} / \mathrm{m}^{2} \text {, if } \\
\text { paclitaxel is not available) + carboplatin } \\
\text { AUC }=5-6 \mathrm{IV}>1 \text { hour, every } 3 \text { weeks }\end{array}$ & China & $\mathrm{P}$ & Recruiting \\
\hline NCT02124421 & $\begin{array}{l}\text { HOT: HIPEC in } \\
\text { Ovarian Cancer as } \\
\text { Initial Treatment }\end{array}$ & $\begin{array}{l}\text { EOC, FTC, } \\
\text { PPC }\end{array}$ & $\begin{array}{l}\text { Experimental arm: CRS/HIPEC using } \\
\text { carboplatin for } 90 \text { minutes with adjuvant } \\
\text { IV chemotherapy with carboplatin and } \\
\text { paclitaxel (Carboplatin AUC } 6 \text {, Paclitaxel } \\
175 \mathrm{mg} / \mathrm{m} 2 \text { ) will be given every } 21 \text { days for a } \\
\text { total of } 6 \text { cycles } \\
\text { Control arm: CRS alone with adjuvant } \\
\text { IV chemotherapy with carboplatin and } \\
\text { paclitaxel (Carboplatin AUC } 6 \text {, Paclitaxel } \\
175 \mathrm{mg} / \mathrm{m}^{2} \text { ) will be given every } 21 \text { days for a } \\
\text { total of } 6 \text { cycles }\end{array}$ & USA & $\mathrm{P}$ & Recruiting \\
\hline
\end{tabular}

Table 4 (continued) 
Table 4 (continued)

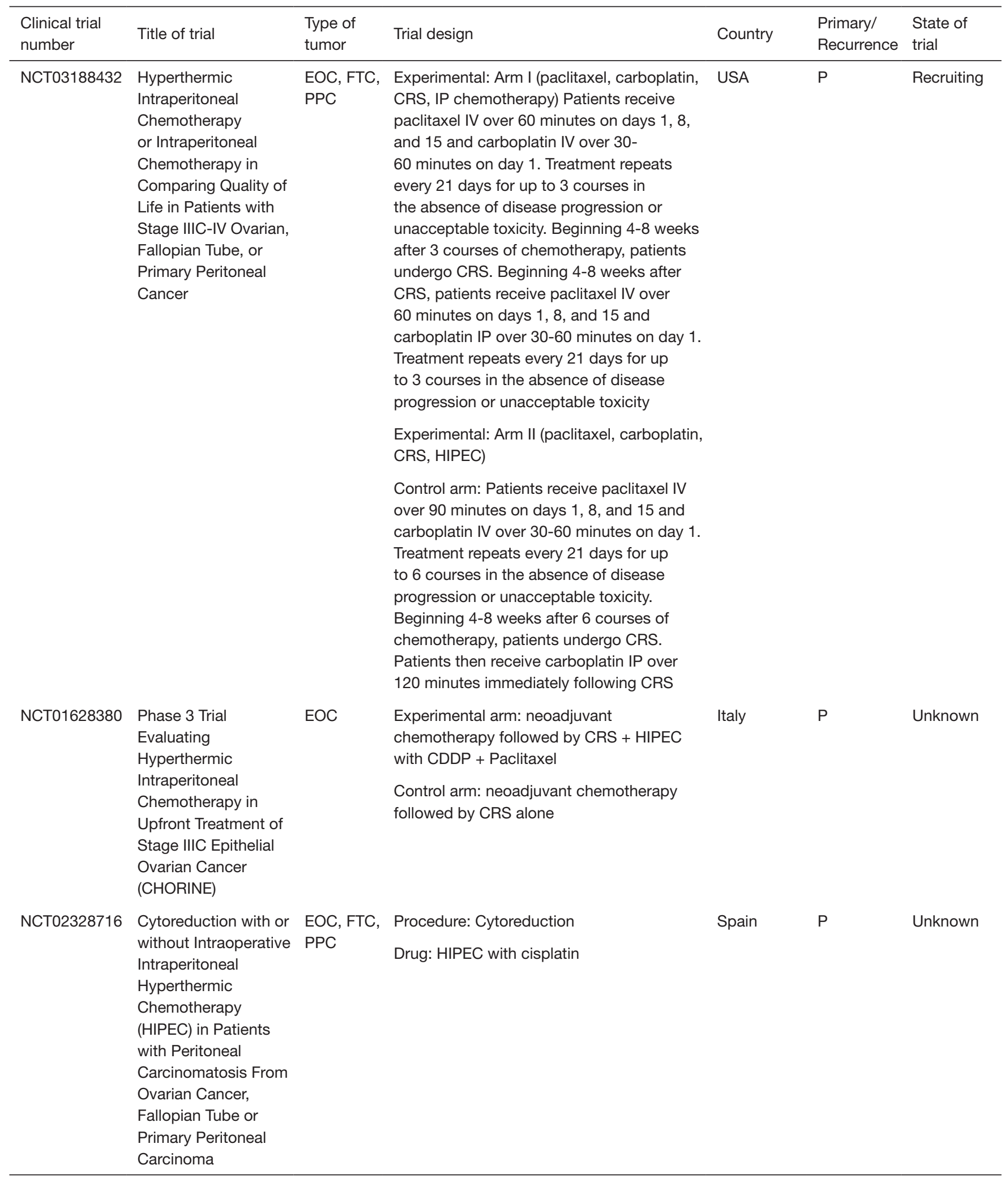

\footnotetext{
Table 4 (continued)
} 
Table 4 (continued)

\begin{tabular}{|c|c|c|c|c|c|c|}
\hline $\begin{array}{l}\text { Clinical trial } \\
\text { number }\end{array}$ & Title of trial & $\begin{array}{l}\text { Type of } \\
\text { tumor }\end{array}$ & Trial design & Country & $\begin{array}{l}\text { Primary/ } \\
\text { Recurrence }\end{array}$ & $\begin{array}{l}\text { State of } \\
\text { trial }\end{array}$ \\
\hline NCT01091636 & $\begin{array}{l}\text { Intraoperative } \\
\text { Hyperthermic } \\
\text { Intraperitoneal } \\
\text { Chemotherapy with } \\
\text { Ovarian Cancer }\end{array}$ & EOC & $\begin{array}{l}\text { Experimental arm: HIPEC in Patients } \\
\text { with ovarian cancer followed by adjuvant } \\
\text { chemotherapy }\end{array}$ & Korea & $\mathrm{P}$ & Unknown \\
\hline NCT00426257 & $\begin{array}{l}\text { Secondary } \\
\text { Debulking Surgery } \\
+/- \text { Hyperthermic } \\
\text { Intraperitoneal } \\
\text { Chemotherapy in } \\
\text { Stage III Ovarian } \\
\text { Cancer (OVHIPEC) }\end{array}$ & EOC & $\begin{array}{l}\text { Experimental arm: Secondary CRS+HIPEC } \\
\text { Control arm: Secondary CRS }\end{array}$ & Netherlands & $\mathrm{R}$ & Completed \\
\hline
\end{tabular}

Table 4 (continued) 
Table 4 (continued)

\begin{tabular}{|c|c|c|c|c|c|c|}
\hline $\begin{array}{l}\text { Clinical trial } \\
\text { number }\end{array}$ & Title of trial & $\begin{array}{l}\text { Type of } \\
\text { tumor }\end{array}$ & Trial design & Country & $\begin{array}{l}\text { Primary/ } \\
\text { Recurrence }\end{array}$ & $\begin{array}{l}\text { State of } \\
\text { trial }\end{array}$ \\
\hline NCT01539785 & $\begin{array}{l}\text { Hyperthermic } \\
\text { Intra-peritoneal } \\
\text { Chemotherapy } \\
\text { (HIPEC) in Ovarian } \\
\text { Cancer Recurrence } \\
\text { (HORSE) }\end{array}$ & EOC & $\begin{array}{l}\text { Experimental arm: CRS followed by HIPEC } \\
\text { in patients with platinum-sensitive first } \\
\text { recurrence of ovarian cancer } \\
\text { Contol arm: vCRS alone }\end{array}$ & Italy & $\mathrm{R}$ & Unknown \\
\hline NCT03220932 & $\begin{array}{l}\text { Cytoreductive Surgery } \\
\text { and HIPEC in First or } \\
\text { Secondary Platinum- } \\
\text { resistant Recurrent } \\
\text { Ovarian Epithelial } \\
\text { Cancer (HIPOVA-01) }\end{array}$ & EOC & $\begin{array}{l}\text { All patients will start with three cycles of CT- } \\
\text { BEV } 15 \mathrm{mg} / \mathrm{kg} \text {, and will then be randomly } \\
\text { Experimental arm: Then one cycle of } \\
\text { monochemotherapy without bevacizumab } \\
\text { is administered and followed by an interval } \\
\text { CRS+HIPEC with adjuvant chemotherapy } \\
\text { and bevacizumab (CT-BEV - } 15 \mathrm{mg} / \mathrm{kg} \text { once } \\
\text { every } 3 \text { weeks) until disease progression } \\
\text { Control arm: Chemotherapy and } \\
\text { bevacizumab (CT-BEV) once every } 3 \text { weeks } \\
\text { from enrollment until disease progression }\end{array}$ & France & $\mathrm{R}$ & $\begin{array}{l}\text { Not yet } \\
\text { recruiting }\end{array}$ \\
\hline NCT03371693 & $\begin{array}{l}\text { Cytoreductive } \\
\text { Surgery (CRS) } \\
\text { Plus Hyperthermic } \\
\text { Intraperitoneal } \\
\text { Chemotherapy } \\
\text { (HIPEC) with } \\
\text { Lobaplatin in } \\
\text { Advanced and } \\
\text { Recurrent Epithelial } \\
\text { Ovarian Cancer } \\
\text { (HIPECOV) }\end{array}$ & EOC & $\begin{array}{l}\text { Experimental arm: CRS+HIPEC and } \\
\text { platinum-based intravenous chemotherapy } \\
\text { A single drug lobaplatin }\left(30 \mathrm{mg} / \mathrm{m}^{2}\right) \text { will be } \\
\text { administered in normal saline via HIPEC } \\
\text { and it will be continued for } 60 \text { minutes in the } \\
\text { hyperthermic phase }\left(41^{\circ} \mathrm{C}-43^{\circ} \mathrm{C}\right) \text {. HIPEC will } \\
\text { be performed at the } 1 \mathrm{st}, 3 \mathrm{rd} \text { and } 5 \text { th day } \\
\text { after CRS. The intravenous chemotherapy } \\
\text { (IVCT) will start from } 7 \text { th- } 14 \text { th day after CRS } \\
\text { Control arm: Only CRS and IVCT. Patients } \\
\text { will receive standard platinum-based } \\
\text { combination doublet chemotherapy for } \\
6-8 \text { cycles after CRS }\end{array}$ & China & $\mathrm{R}$ & $\begin{array}{l}\text { Active, not } \\
\text { recruiting }\end{array}$ \\
\hline
\end{tabular}

Eoc, epithelial ovarian cancer; P, primary; R, recurrence; CRS, cytoreductive surgery; HIPEC, hyperthermic intraperitoneal chemotherapy; FTC, Fallopian tube cancer; PPC, primary peritoneal carcinoma; IVCT, intravenous chemotherapy; underline cells, recruiting. 
Table 5 Retrospective observational studies focus on intraperitoneal chemotherapy for recurrent EOC

\begin{tabular}{|c|c|c|c|c|c|c|c|c|c|c|c|c|c|c|c|}
\hline $\begin{array}{l}\text { First authors } \\
\text { (ref.) }\end{array}$ & Year & $\begin{array}{l}\text { Case- } \\
\text { control or } \\
\text { cohort }\end{array}$ & $\begin{array}{l}\text { Primitive/ } \\
\text { Recurrence }\end{array}$ & $\begin{array}{l}\text { FlGO (Inclusion of } \\
\text { persistent EOC } \text { EC? }^{\delta} \text { ) }\end{array}$ & $\begin{array}{l}\text { IP therapy } \\
\text { technique }\end{array}$ & No. of pts & $\begin{array}{l}\text { Treatment and Chemotherapy } \\
\text { regimen^ }\end{array}$ & $\begin{array}{l}\text { Platinum-Sensitive } \\
\text { pts }\end{array}$ & CC0-1 n (\%) & \multicolumn{2}{|c|}{ Median OS (months) [5y-OS\%] } & \multicolumn{2}{|c|}{$\begin{array}{l}\text { Median DFS or PFS } \\
\text { (months) [5y-DFS\%] }\end{array}$} & $\begin{array}{l}\text { Morbidity (3-4 } \\
\text { grade/severe) \% }\end{array}$ & Mortality \% \\
\hline \multicolumn{16}{|l|}{ HIPEC } \\
\hline Amira (33) & 2018 & Ca-Co & rEOC & $-($ no) & HIPEC & $15 \mathrm{ca}$ & CRS + HIPEC with Cis $100 \mathrm{mg} / \mathrm{m}^{2}$ & $100 \%$ Platinum-S & - & \multicolumn{2}{|l|}{36} & \multicolumn{2}{|l|}{6} & - & 13.3 \\
\hline $\begin{array}{l}\text { Arjona- } \\
\text { Sanchez (34) }\end{array}$ & 2018 & Cohort & rEOC & $\begin{array}{l}\text { IIIC-IV with down- } \\
\text { staging after } \\
\text { NACT (no) }\end{array}$ & HIPEC & 100 & $\begin{array}{l}\text { NACT with IV Carboplatin + Taxol (or } \\
\text { Doxo for platinum-resistant) + HIPEC } \\
\text { with Paclitaxel } 60 \mathrm{mg} / \mathrm{m}^{2}\end{array}$ & $\begin{array}{l}98 \% \text { Platinum-S, } 2 \% \\
\text { Platinum-R }\end{array}$ & $\begin{array}{l}\text { CC-0: } 92(92) \\
\text { CC-1: } 8(8)\end{array}$ & $48.2[64]$ & $\begin{array}{l}\text { CC-0: } 49.3 \text { [59], } \\
\text { CC-1: } 31.6[35] . \\
\text { Negative lymph } \\
\text { nodes: } 49.3 \text { [58]; } \\
\text { Positive lymph } \\
\text { nodes: } 42.6[46]\end{array}$ & \multicolumn{2}{|l|}{-} & 16 & 0 \\
\hline \multirow[t]{2}{*}{ Baiocchi (35) } & 2016 & $\mathrm{Ca}-\mathrm{Co}$ & rEOC & I-IV (no) & HIPEC & $50 \mathrm{ca}$ & $\begin{array}{l}\text { CRS + HIPEC with MMC } 10 \mathrm{mg} / \mathrm{m}^{2}+ \\
\text { Cis }\left(50 \mathrm{mg} / \mathrm{m}^{2}\right): 15 \mathrm{pts} ; \text { Cis }\left(50 \mathrm{mg} / \mathrm{m}^{2}\right)+ \\
\text { Doxo: } 8 \mathrm{pts} \text { O Oxa: } 3 \mathrm{pts} \text {; Cis: } 3 \mathrm{pts}\end{array}$ & $100 \%$ Platinum-S & $\begin{array}{l}\text { CC-0: } 60(77.9) \\
\text { CC-1: } 9(11.7) \\
\text { CC-2,3: } 8(10.4)\end{array}$ & $58.3[49.7]$ & & \multirow{2}{*}{\multicolumn{2}{|c|}{15.8}} & $69.0(34.5)$ & 0 \\
\hline & & & & & & 29 co & CRS & & & $59.3[49.5]$ & & & & $42.6(10.6)$ & 4 \\
\hline Bakrin (36) & 2012 & Cohort & rEOC & - (yes) & HIPEC & 246 & $\begin{array}{l}\text { Platinum-based CT before surgery + } \\
\text { CRS + HIPEC with Cis alone or with } \\
\text { Doxo/Mito }\end{array}$ & $\begin{array}{l}\text { Platinum-S } 184 \text { (74.8), } \\
\text { Platinum-R } 62 \text { (25.2) }\end{array}$ & $\begin{array}{l}\text { CC-0,1: } 247 \text { (92.2), } \\
\text { CC-2,3: } 21 \text { (7.8) }\end{array}$ & $48.9[35]$ & $\begin{array}{l}\text { Platinum-R:48, } \\
\text { Platinum-S: } 52\end{array}$ & \multicolumn{2}{|l|}{$12.8[9]$} & (11.6) & 0.37 \\
\hline Carrabin (37) & 2010 & Cohort & rEOC & IIIC (yes) & HIPEC & $\begin{array}{l}18 \text { ( } 8 \text { recur-rent, } 10 \\
\text { persistent) }\end{array}$ & CRS+HIPEC with Oxa 460 mg/m² & $\begin{array}{l}\text { Platinum-S } 7 \text { (87.5), } \\
\text { Platinum-R: } 1 \text { (12.5) }\end{array}$ & $\begin{array}{l}\text { CC-0: } 16 \text { (88.9), CC- } \\
1: 2(11.1)\end{array}$ & \multicolumn{2}{|c|}{$\begin{array}{l}\text { Not reached, 2y-OS 92\%, } \\
\text { 3y-OS } 83 \%\end{array}$} & \multicolumn{2}{|l|}{11.3} & (55.6) & 0 \\
\hline \multirow[t]{2}{*}{$\begin{array}{l}\text { Cascales- } \\
\text { Campos (38) }\end{array}$} & 2015 & Ca-Co & rEOC & I-IV (no) & HIPEC & Са 32 & $\begin{array}{l}\text { Pre-op Platinum + Taxanes EV if } \\
\text { unresectable }+ \text { CRS }+ \text { HIPEC with } \\
\text { Paclitaxel } 60 \mathrm{mg} / \mathrm{m}^{2}\end{array}$ & $100 \%$ Platinum-S & CC-0 $54(100)$ & \multirow{2}{*}{\multicolumn{2}{|c|}{ - }} & \multirow{2}{*}{\multicolumn{2}{|c|}{ 3y DFS: 45}} & $28(21)$ & 0 \\
\hline & & & & & & Co 22 & $\begin{array}{l}\text { Pre-op Platinum + Taxanes EV if } \\
\text { unresectable + CRS }\end{array}$ & & & & & & & $23(14)$ & \\
\hline $\begin{array}{l}\text { Chatzigeorgiou } \\
\text { (39) }\end{array}$ & 2003 & Cohort & rEOC & - & HIPEC & 20 & CRS+HIPEC with Cis $50-70 \mathrm{mg} / \mathrm{m}^{2}$ & $\begin{array}{l}30 \% \text { Platinum-S, } 70 \% \\
\text { Platinum-R }\end{array}$ & $\begin{array}{l}\text { Residual tumor (RT). } \\
<1.5 \mathrm{cm:} 12(60) \\
>1.5 \mathrm{~cm}: 8(40)\end{array}$ & \multicolumn{2}{|c|}{$\begin{array}{l}\text { RT <1.5 cm: } 29, \mathrm{RT}>1.5 \mathrm{~cm}: 7 . \\
\text { Platinum-S: } 27 \text {, Platinum-R: } 9\end{array}$} & \multicolumn{2}{|l|}{ - } & 15 & 5 \\
\hline Cianci (40) & 2019 & Cohort & $\begin{array}{l}\text { rEOC (previous } \\
\text { CRS+HIPEC) }\end{array}$ & $-(\mathrm{no})$ & $\begin{array}{l}\text { Tertiary } \\
\text { HIPEC }\end{array}$ & 12 & $\begin{array}{l}\text { Tertiary CRS + HIPEC with Oxa } \\
360 \mathrm{mg} / \mathrm{m}^{2} \text { or Cis } 75 \mathrm{mg} / \mathrm{m}^{2}\end{array}$ & $100 \%$ Platinum-S & $100 \% \mathrm{RT}=0$ & \multicolumn{2}{|l|}{ Mean OS 99} & \multicolumn{2}{|l|}{28} & $24.9(8.3)$ & 0 \\
\hline Classe (41) & 2015 & Cohort & $\begin{array}{l}\text { rEOC (first } \\
\text { relapse) }\end{array}$ & (yes) & HIPEC & 314 & $\begin{array}{l}\text { (Ev. second-line IV CT) + CRS + HIPEC } \\
\text { with Cis }\end{array}$ & $\begin{array}{l}\text { Platinum-R } 52.9 \% \\
\text { Platinum-S } 47.1 \%\end{array}$ & $\begin{array}{l}\text { CC-0: } 248 \text { (79) } \\
\text { CC-1.2: } 66(21)\end{array}$ & [38] & $\begin{array}{l}\text { Platinum-S: } 42 \\
\text { Platinum-R: } 51 \\
\text { CC-0: } 54 \\
\text { CC-1.2: } 36\end{array}$ & [14] & $\begin{array}{l}\text { Platinum-S: } 13 \\
\text { Platinum-R: } 14 \\
\text { CC-0: } 15 \\
\text { CC-1,2: } 10\end{array}$ & (30.9) & 1 \\
\hline \multirow[t]{2}{*}{ Cotte (42) } & 2007 & Cohort & rEOC & (yes) & HIPEC & $\begin{array}{l}\text { Persistence of disease }+ \\
\text { Platinum-R recurrence } 16\end{array}$ & CRS + HIPEC with Cis $20 \mathrm{mg} / \mathrm{m}^{2} / \mathrm{L}$ & $\begin{array}{l}\text { Platinum-R } 19.8 \% \\
\text { Platinum-S } 80.2 \%\end{array}$ & $\begin{array}{l}\text { CC-0: } 45(55.5) \\
\text { CC-1: } 20(24.7)\end{array}$ & 24.3 & $\begin{array}{l}\mathrm{PCl}<12: 37.6 \\
\mathrm{PCl}>12: 13.1\end{array}$ & 8.0 & $\begin{array}{l}\mathrm{PCl}<12: 12.8 \\
\text { PCI>12: 2.4; }\end{array}$ & (13.6) & 2.5 \\
\hline & & & & & & Platinum-S recurrence 65 & & & CC-2: $16(19.8)$ & 28.4 & $\begin{array}{l}\text { CC-0: 54.9, CC-1: } \\
\text { 17.0, CC-2: } 5.1\end{array}$ & 8.5 & CC-0: 47.8 & & \\
\hline Delotte (43) & 2014 & Cohort & $\mathrm{rEOC}>70 \mathrm{y}$ & $-(\mathrm{no})$ & HIPEC & 15 & $\begin{array}{l}\text { CRS+HIPEC with Cis } 50 \mathrm{mg} / \mathrm{m}^{2}+\text { Doxo } \\
15 \mathrm{mg} / \mathrm{m}^{2}\end{array}$ & - & $\begin{array}{l}\text { CC-0: } 9(60), \\
\text { CC-1: } 6(40)\end{array}$ & 35 & & 15.6 & & (20) & 0 \\
\hline Deraco (44) & 2001 & Cohort & rEOC & I-IV (yes) & HIPEC & 27 & $\begin{array}{l}\text { CRS+HIPEC with Cis } 25 \mathrm{mg} / \mathrm{m}^{2} / \mathrm{L}+ \\
\text { MMC } 3.3 \mathrm{mg} / \mathrm{m}^{2} / \mathrm{L}\end{array}$ & - & $\begin{array}{l}\text { CC-0: } 15 \text { (55), CC-1: } \\
4 \text { (15), CC-2: } 3 \text { (11), } \\
\text { CC-3: } 5 \text { (19) }\end{array}$ & [2yOS: 55\%] & $\begin{array}{l}\text { CC-0,1: 20.3 [2yOS } \\
77 \%], \text { CC-2,3: } 4.3 \\
\text { [2yOS 0\%] }\end{array}$ & $21.8[2 y \mathrm{D}$ & DFS: 21\%] & 11 & 4 \\
\hline
\end{tabular}

Table 5 (contimued) 


\begin{tabular}{|c|c|c|c|c|c|c|c|c|c|c|c|c|c|}
\hline $\begin{array}{l}\text { First authors } \\
\text { (ref.) }\end{array}$ & Year & $\begin{array}{l}\text { Case- } \\
\text { control or } \\
\text { cohort }\end{array}$ & $\begin{array}{l}\text { Primitive/ } \\
\text { Recurrence }\end{array}$ & $\begin{array}{l}\text { FIGO (Inclusion of } \\
\text { persistent EOC? ?) }\end{array}$ & $\begin{array}{l}\text { IP therapy } \\
\text { technique }\end{array}$ & No. of pts & $\begin{array}{l}\text { Treatment and Chemotherapy } \\
\text { regimen^ }\end{array}$ & $\begin{array}{l}\text { Platinum-Sensitive } \\
\text { pts }\end{array}$ & cco-1 n (\%) & Median OS (months) [5y-OS\%] & $\begin{array}{l}\text { Median DFS or PFS } \\
\text { (months) [5y-DFS\%] }\end{array}$ & $\begin{array}{l}\text { Morbidity (3-4 } \\
\text { grade/severe) } \%\end{array}$ & Mortality \% \\
\hline Deraco (45) & 2012 & Cohort & rEOC & $\mathrm{I}-\mathrm{IV}(\mathrm{no})$ & HIPEC & 56 & $\begin{array}{l}\text { CRS +HIPEC with Cis } 42 \mathrm{mg} / \mathrm{L}+\text { Doxo } \\
15 \mathrm{mg} / \mathrm{L} \text { or Cis } 25 \mathrm{mg} / \mathrm{L} \mathrm{m}^{2}+\mathrm{MMC} \\
3.3 \mathrm{mg} / \mathrm{L} / \mathrm{m}^{2}\end{array}$ & $\begin{array}{l}\text { Platinum-S 58.9\% } \\
\text { Platinum-R } 23.1 \%\end{array}$ & $\begin{array}{l}\text { CC-0: } 46(83.9) \\
\text { CC-1: } 7(12.5) \\
\text { CC-2: } 1(1.8)\end{array}$ & 25.7 [23] & $10.8[7]$ & (26.3) & 5.3 \\
\hline Fagotti (46) & 2009 & Cohort & rEOC & I-IV (yes) & HIPEC & 25 & $\begin{array}{l}\text { CRS+HIPEC with Oxa } 460 \mathrm{mg} / \mathrm{m}^{2}+ \\
\text { post-op IV Oxa + Docetaxel }\end{array}$ & Platinum-S 100\% & $\begin{array}{l}\text { CC-0: } 23(92) \\
\text { CC-1: } 2(8)\end{array}$ & - & 10 & (28) & 0 \\
\hline \multirow[t]{2}{*}{ Fagotti (47) } & 2012 & Ca-Co & rEOC & I-IV (yes) & HIPEC & $30 \mathrm{Ca}$ & $\begin{array}{l}\text { CRS+HIPEC with Oxa } 469 \mathrm{mg} / \mathrm{m}^{2}+ \\
\text { post-op IV platinum CT }\end{array}$ & Platinum-S 100\% & $\begin{array}{l}\text { CC-0: } 29(96.7) \\
\text { CC-1: } 1(3.3)\end{array}$ & [68.4] & 26 & (34.8) & 0 \\
\hline & & & & & & $37 \mathrm{Co}$ & CRS+IV CT (35\%) or IV CT (65\%) & & - & [42.7] & 15 & & \\
\hline Fagotti (48) & 2014 & Cohort & $\begin{array}{l}\text { rEOC (single } \\
\text { nodule) }\end{array}$ & Single nodule (no) & $\begin{array}{l}\text { Laparoscopic } \\
\text { HIPEC }\end{array}$ & 10 & $\begin{array}{l}\text { Laparoscopic/robotic CRS+HIIPEC with } \\
\text { Oxa } 469 \mathrm{mg} / \mathrm{m}^{2} \text { or Cis } 75 \mathrm{mg} / \mathrm{m}^{2}+\text { post- } \\
\text { op IV carboplatin-paclitaxel CT }\end{array}$ & Platinum-S 100\% & CC-0: $100 \%$ & - & - & 10 & 0 \\
\hline Fahim (49) & 2018 & Cohort & rEOC & (no) & HIPEC & 9 & CRS +HIPEC with Cis $200 \mathrm{mg} / \mathrm{m}^{2}$ & Platinum-S 100\% & - & 42 & Not reached & - & 22.2 \\
\hline Furet (50) & 2013 & Cohort & rEOC & - & HIPEC & 17 & $\begin{array}{l}\text { CRS+HIPEC with Oxa } 460 \mathrm{mg} / \mathrm{m}^{2} \\
+ \text { IV } 5 F U 400 \mathrm{mg} / \mathrm{m}^{2} \text { or Carbo } 400- \\
1200 \mathrm{mg} / \mathrm{m}^{2}\end{array}$ & - & CC-0: 16 (94) & mean OS: 30.5 & 11.9 & $47(17.6)$ & 0 \\
\hline $\begin{array}{l}\text { Gomez-Ruiz } \\
\text { (51) }\end{array}$ & 2019 & Cohort & rEOC & I-IV (-) & HIPEC & 64 & $\begin{array}{l}\text { CRS+HIIPEC with Paclitaxel } 60 \mathrm{mg} / \mathrm{m}^{2} \text { or } \\
\text { Cis } 75 \mathrm{mg} / \mathrm{m}^{2}\end{array}$ & Platinum-S 100\% & $\begin{array}{l}\text { CC-0: } 57 \text { (89) } \\
\text { CC-1: } 7(11)\end{array}$ & - & $17[30]$ & - & - \\
\hline Helm (52) & 2007 & Cohort & rEOC & IIIC-IV & HIPEC & 18 & $\begin{array}{l}\text { CRS+HIPEC with Cis } 100 \mathrm{mg} / \mathrm{m}^{2} \text { or } \\
\text { MMC } 40 \mathrm{mg}\end{array}$ & - & $\begin{array}{l}\text { CC-0: } 11(61.1) \\
\text { CC-1: } 6 \text { (33.4) } \\
\text { CC-2: } 1(5.5)\end{array}$ & $31, \mathrm{RT}<2 \mathrm{~mm}: 31, \mathrm{RT}>2 \mathrm{~mm}: 8$ & 10 & $100(72)$ & 5.5 \\
\hline $\begin{array}{l}\text { Konigsrainer } \\
\text { (53) }\end{array}$ & 2011 & Cohort & rEOC & IIIIb-IV (-) & HIPEC & 31 & CRS+HIPEC with Cis $50 \mathrm{mg} / \mathrm{m}^{2}$ & - & $\begin{array}{l}\text { CC-0: } 20(65) \\
\text { CC-1: } 8(25) \\
\text { CC-2: } 3(10)\end{array}$ & [3y-OS 50\%] & [3y-DFS 15\%] & 23 & 0 \\
\hline \multirow[t]{2}{*}{$\begin{array}{l}\text { Konigsrainer } \\
\text { (54) }\end{array}$} & 2014 & Cohort & rEOC & I-IV (-) & HIPEC & CC-0,1: 62 & CRS+HIPEC with Cis $50 \mathrm{mg} / \mathrm{m}^{2}$ & $\begin{array}{l}\text { Platinum-R } 13 \% \\
\text { Platinum-S } 87 \%\end{array}$ & $\begin{array}{l}\text { CC-0: } 47(52) \\
\text { CC-1: } 15(17)\end{array}$ & 35 & - & $45(26)$ & 0 \\
\hline & & & & & & CC-2,3: 28 & & & $\begin{array}{l}\text { CC-2: } 5(6) \\
\text { CC-3: } 23(26)\end{array}$ & 14 & & $36(11)$ & 0 \\
\hline \multirow[t]{2}{*}{ Le Brun (55) } & 2014 & Ca-Co & rEOC & I-IV (yes) & HIPEC & Сa 23 & CRS+HIPEC with Cis, Eloxatin, MMC & Platinum-S 100\% & CC-0: $42(100)$ & {$[4 y-0 S 75.6]$} & - & - & - \\
\hline & & & & & & Co 19 & CRS & & & [4y-OS 19.4] & & & \\
\hline \multirow[t]{3}{*}{ Marocco (56) } & 2016 & Ca-Co & rEOC & I-IV (yes) & HIPEC & 16 & IV platinum-based CT & Platinum-S 100\% & - & 35.6 & 13.2 & $0(0)$ & 0 \\
\hline & & & & & & 11 & CRS + IV platinum-based CT & & CC-0 100\% & Not reached & 23 & $36(0)$ & 0 \\
\hline & & & & & & 19 & $\begin{array}{l}\text { IV platinum-based CT + CRS + HIPEC } \\
\text { with Cis } 100 \mathrm{mg} / \mathrm{m}^{2}+\text { Doxo } 15.2 \mathrm{mg} / \mathrm{L}\end{array}$ & & & 51.5 & 19.9 & $32(16)$ & 0 \\
\hline \multirow[t]{2}{*}{$\begin{array}{l}\text { Munoz- } \\
\text { Casares (57) }\end{array}$} & 2009 & Ca-Co & rEOC & III & HIPEC & $14 \mathrm{Ca}$ & $\begin{array}{l}\text { CRS+HIPEC with Paclitaxel } 60 \mathrm{mg} / \mathrm{m}^{2}+ \\
\text { post-op IV CT }\end{array}$ & - & $\begin{array}{l}\text { CC-0: } 16(61.5) \\
\text { CC-1: } 10(38.5)\end{array}$ & [57] [for CC-0 67] & Mean DFS 48 & $29(14)$ & 0 \\
\hline & & & & & & $12 \mathrm{Co}$ & CRS + post-op IV CT & & & [17] [for CC-0 29] & Mean DFS 24 & $25(25)$ & 0 \\
\hline Petrillo (58) & 2016 & Cohort & rEOC & I-IV & HIPEC & 70 & $\begin{array}{l}\text { CRS + HIPEC with Oxa } 460 \mathrm{mg} / \mathrm{m}^{2} \text { or } \\
\text { Cis } 75 \mathrm{mg} / \mathrm{m}^{2}\end{array}$ & Platinum-S 100\% & $\begin{array}{l}\text { CC-0: } 62(88.6) \\
\text { CC-1: } 8(11.4)\end{array}$ & - & 27 & $35.7(8.5)$ & 0 \\
\hline
\end{tabular}

Table 5 (contimued) 


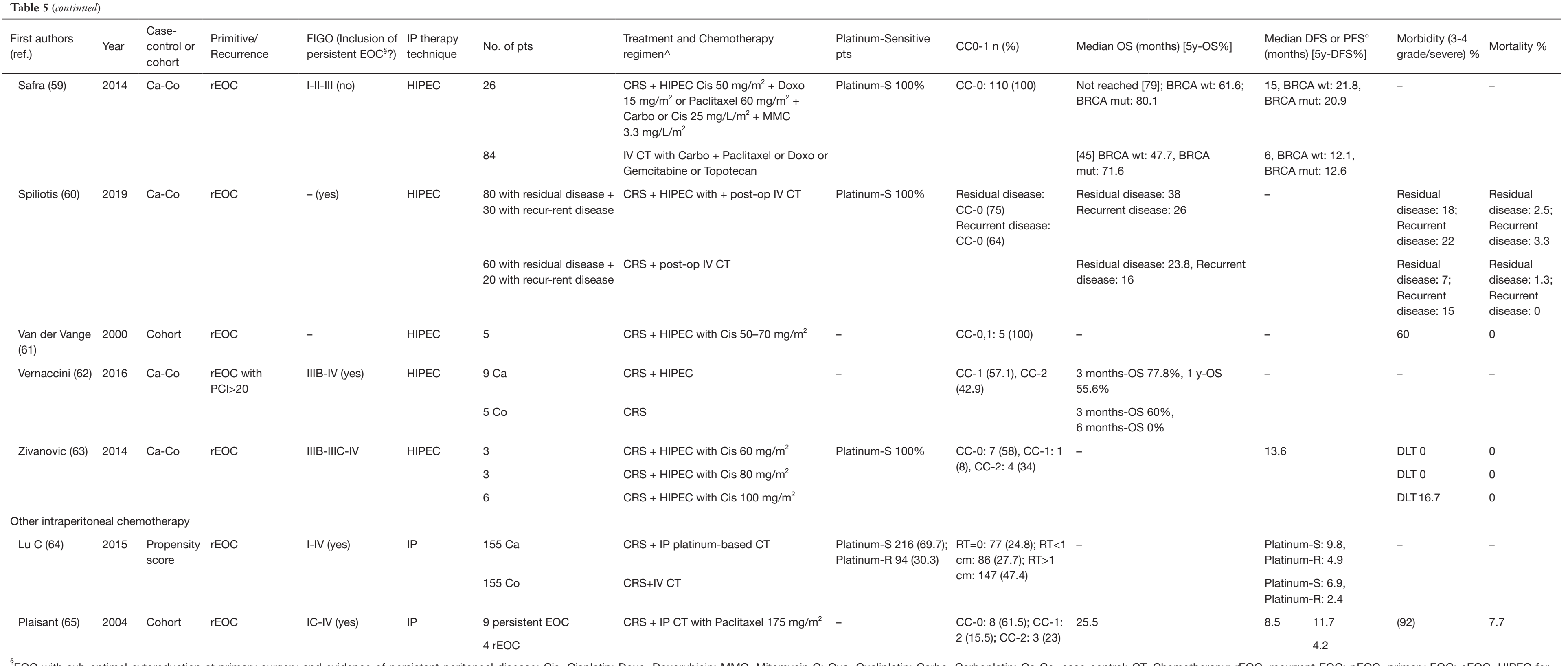

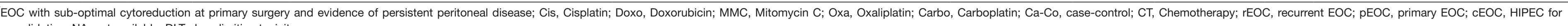
consolidation. NA, not available; DLT, dose limiting toxicity. 
Table 6 Prospective studies focus on intraperitoneal chemotherapy for recurrent EOC

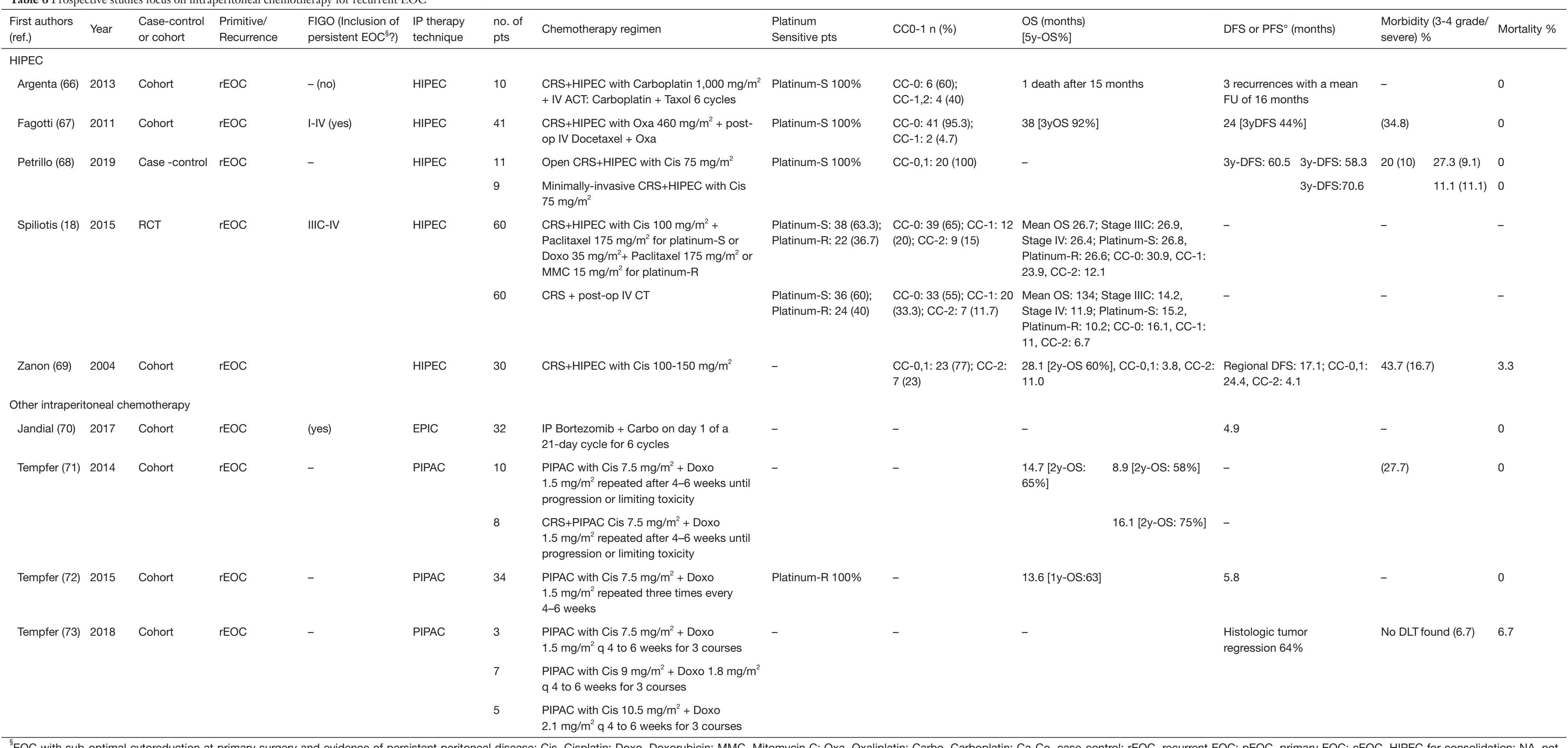

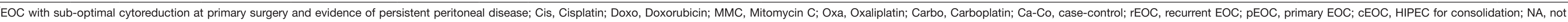
available; DLT, dose limiting toxicity. 
Table 7 Retrospective observational studies focus on intraperitoneal chemotherapy for primary and recurrent EOC

\begin{tabular}{|c|c|c|c|c|c|c|c|c|c|c|c|c|c|c|c|}
\hline $\begin{array}{l}\text { First authors } \\
\text { (ref.) }\end{array}$ & Year & $\begin{array}{l}\text { Case-control } \\
\text { or cohort }\end{array}$ & $\begin{array}{l}\text { Primitive/ } \\
\text { Recurrence }\end{array}$ & $\begin{array}{l}\text { FIGO (Inclusion of } \\
\text { persistent EOC }{ }^{\uparrow} \text { ?) }\end{array}$ & $\begin{array}{l}\text { IP therapy } \\
\text { technique }\end{array}$ & no. of pts & Treatment and Chemotherapy regimen & $\begin{array}{l}\text { Platinum- } \\
\text { Sensitive pts }\end{array}$ & CC0-1 n (\%) & \multicolumn{2}{|c|}{ Median OS (months) [5y-OS\%] } & \multicolumn{2}{|c|}{$\begin{array}{l}\text { Median DFS or PFS }(\text { months) } \\
\text { [5y-DFS\%] }\end{array}$} & $\begin{array}{l}\text { Morbidity (3-4 } \\
\text { grade/severe) \% }\end{array}$ & $\begin{array}{l}\text { Mortality } \\
\%\end{array}$ \\
\hline \multirow[t]{2}{*}{$\begin{array}{l}\text { Arjona- } \\
\text { Sanchez (74) }\end{array}$} & \multirow[t]{2}{*}{2017} & \multirow[t]{2}{*}{ Cohort } & pEOC & IIIC-IV & HIPEC & 320 & $\begin{array}{l}\text { CRS+HIPEC with Paclitaxel } 60 \mathrm{mg} / \mathrm{m}^{2} \text { or } \\
\text { Cis } 75 \mathrm{mg} / \mathrm{m}^{2}\end{array}$ & - & $\begin{array}{l}\text { CC-0: } 500 \text { (95) } \\
\text { CC-1,2: } 27 \text { (5) }\end{array}$ & \multicolumn{2}{|c|}{$\begin{array}{l}\text { 1997-2004: } 38 \text { [37]; 2005-2009: [48]; } \\
\text { 2010-2012: } 57 \text { [49]; 2012-2016: 43 }\end{array}$} & \multicolumn{2}{|c|}{$\begin{array}{l}\text { 1997-2004: 25; 2005-2009: -; 2010- } \\
\text { 2012: 24; 2012-2016: } 27\end{array}$} & (15) & 2 \\
\hline & & & rEOC & & & 207 & & & & \multicolumn{2}{|c|}{$\begin{array}{l}\text { 1997-2004: } 57 \text { [51]; 2005-2009: [48]; } \\
\text { 2010-2012: } 55 \text { [47]; 2012-2016: } 35\end{array}$} & \multicolumn{2}{|c|}{$\begin{array}{l}\text { 1997-2004: 31; 2005-2009: -; 2010- } \\
\text { 2012: 19; 2012-2016: } 27\end{array}$} & & \\
\hline \multirow[t]{3}{*}{ Bakrin (75) } & \multirow[t]{3}{*}{2013} & \multirow[t]{3}{*}{ Cohort } & $\mathrm{pEOC}$ & - & HIPEC & 36 & (ev. NACT) + CRS + HIPEC with Cis, MMC, & - & CC-0: $423(74.3)$ & \multirow{2}{*}{\multicolumn{2}{|c|}{35.4}} & \multirow{2}{*}{\multicolumn{2}{|c|}{11.8}} & (31.3) & 0.8 \\
\hline & & & CEOC & & & 56 & Doxo, Oxa & & & & & & & & \\
\hline & & & rEOC & & & 477 & & $\begin{array}{l}\text { Platinum-S: } \\
46.8 \%, \\
\text { Platinum-R: } \\
51.8 \%\end{array}$ & & \multicolumn{2}{|c|}{45.7} & \multicolumn{2}{|l|}{-} & & \\
\hline \multirow[t]{2}{*}{ Barakat (76) } & \multirow[t]{2}{*}{2002} & \multirow[t]{2}{*}{ Cohort } & rEOC & II-IV (yes) & IP & 322 & $\begin{array}{l}\text { Platinum-based combination IP CT at the } \\
\text { time of second-look assessment }\end{array}$ & - & - & \multicolumn{2}{|c|}{$\begin{array}{l}\text { Microscopic disease: } 57.6 \text {; Disease } \\
>1 \mathrm{~cm}: 14.4 \text {; Disease }<1 \mathrm{~cm}: 39.6\end{array}$} & \multicolumn{2}{|c|}{$\begin{array}{l}\text { Microscopic disease: } 26.3 \text {, Gross } \\
\text { disease: } 16.3\end{array}$} & 22.6 & - \\
\hline & & & CEOC & & & 89 & & & & \multicolumn{2}{|l|}{104.4} & \multicolumn{2}{|l|}{32} & & \\
\hline \multirow{3}{*}{$\begin{array}{l}\text { Cascales- } \\
\text { Campos } \\
\text { (770) }\end{array}$} & \multirow[t]{3}{*}{2014} & \multirow[t]{3}{*}{ Cohort } & PEOC & IIIB-IV & HIPEC & 22 & $\begin{array}{l}\text { CRS+HIPEC with Paclitaxel } 60 \mathrm{mg} / \mathrm{m}^{2} \text { or } \\
\text { Cis } 75 \mathrm{mg} / \mathrm{m}^{2}\end{array}$ & $\begin{array}{l}\text { Platinum-S } \\
100 \%\end{array}$ & $\begin{array}{l}\text { CC-0: } 73(80.2) \\
\text { CC-1: } 18(19.8)\end{array}$ & \multirow{3}{*}{\multicolumn{2}{|c|}{-}} & \multirow{3}{*}{\multicolumn{2}{|c|}{-}} & $27(12)$ & 0 \\
\hline & & & & & & 38 & $\begin{array}{l}\text { NACT }+ \text { CRS }+ \text { HIPEC with Paclitaxel } \\
60 \mathrm{mg} / \mathrm{m}^{2} \text { or Cis } 75 \mathrm{mg} / \mathrm{m}^{2}\end{array}$ & & & & & & & & \\
\hline & & & rEOC & & & 31 & $\begin{array}{l}\text { CRS+HIPEC with Paclitaxel } 60 \mathrm{mg} / \mathrm{m}^{2} \text { or } \\
\text { Cis } 75 \mathrm{mg} / \mathrm{m}^{2}\end{array}$ & & & & & & & & \\
\hline Cripe (78) & 2015 & Cohort & $\begin{array}{l}\text { pEOC, rEOC, } \\
\text { cEOC }\end{array}$ & $\mathrm{I}-\mathrm{IV}(\mathrm{no})$ & HIPEC & $\begin{array}{l}24 \text { rEOC, } 6 \text { pEOC, } \\
2 \text { cEOC }\end{array}$ & $\begin{array}{l}\text { CRS + HIPEC with Oxa } 460 \mathrm{mg} / \mathrm{m}^{2}+\text { IV } \\
\text { 5-FU or MMC } 20 \mathrm{mg} / \mathrm{m}^{2} \text { or Oxa } \\
460 \mathrm{mg} / \mathrm{m}^{2} \text { or Cis } 75 \mathrm{mg} / \mathrm{m}^{2} \text { or Cis } \\
40 \mathrm{mg} / \mathrm{m}^{2} \text { Doxo } 12 \mathrm{mg} / \mathrm{m}^{2} \text { or Doxo } \\
15 \mathrm{mg} / \mathrm{m}^{2}\end{array}$ & $\begin{array}{l}\text { Platinum-S } \\
100 \%\end{array}$ & $\begin{array}{l}\text { CC-0: } 29 \text { (90.6), CC-1: } \\
2 \text { (6.3), CC-2: } 1 \text { (3.1) }\end{array}$ & - & & - & & (65.6) & 0 \\
\hline \multirow[t]{2}{*}{$\begin{array}{l}\text { Di Giorgio } \\
\text { (79) }\end{array}$} & \multirow[t]{2}{*}{2017} & \multirow[t]{2}{*}{ Cohort } & pEOC & III-IV & HIPEC & 226 & \multirow[t]{2}{*}{$\begin{array}{l}\text { CRS+HIPEC with Oxa } 460 \mathrm{mg} / \mathrm{m}^{2} \text { or Cis } 75 \\
\mathrm{mg} / \mathrm{m}^{2} \text { or Cis } 75 \mathrm{mg} / \mathrm{m}^{2} \text { Doxo/Paclitaxel/ } \\
\mathrm{MMC}\end{array}$} & - & CC-0: $160(70.8)$ & $52.4[4.4]$ & $\begin{array}{l}54.2 \\
\text { PDS: } 61.2 \\
\text { prNACT + IDS: } 58 \\
\text { nrNACT + IDS: } 34.7 \\
\text { crNACT + IDS: NR }\end{array}$ & $16.6[19.7]$ & $\begin{array}{l}20,2 \\
\text { PDS:29,9 } \\
\text { prNACT + IDS: } 15 \\
\text { nrNACT + IDS: } 14.5 \\
\text { crNACT + IDS: NR }\end{array}$ & $44.2(17.4)$ & 2.5 \\
\hline & & & rEOC & & & 285 & & & CC-0: 211 (74) & & $\begin{array}{l}\text { 46.8. fr PFS > } 12 \text { months: } \\
\text { 96.3; fr PFR < } 12 \text { months: } \\
\text { 35.7; fr PFS }>12 \text { months } \\
\text { further CT: } 48.3 \text {; more CRS } \\
\text { and more chemo-therapy } \\
\text { before HIPEC: } 35.7\end{array}$ & & $\begin{array}{l}\text { 15.2. fr PFS }>12 \text { months: } \\
\text { 17.5; fr PFR }<12 \text { months: } \\
\text { 12.4; fr PFS }>12 \text { months } \\
\text { further CT: } 14.2 \text {; more CRS } \\
\text { and more chemo-therapy } \\
\text { before HIPEC: } 15.4\end{array}$ & & \\
\hline Frenel (80) & 2011 & Cohort & pEOC & IIIC-IV & HIPEC & 19 & (ev NACT) + CRS+HIPEC with Oxa & - & CC-0: 31 (100) & - & & $13.2[1 \mathrm{y}-\mathrm{DFs}$ & 59.3\%] & (29) & 0 \\
\hline & & & rEOC & & & 12 & $360-460 \mathrm{mg} / \mathrm{m}^{-3}$ & & & & & $14.3[1 \mathrm{y}-\mathrm{DFS}$ & 54.4\%] & & \\
\hline Helm (20) & 2010 & Cohort & pEOC & II-IV & HIPEC & 45 & $\begin{array}{l}\text { (ev NACT) + CRS+HIPEC with Carbo or Cis } \\
\text { or Cis + Doxo or Oxa or MMC or Carbo + }\end{array}$ & $\begin{array}{l}\text { Platinum-S: } \\
61.3 \% \text {, }\end{array}$ & $\begin{array}{l}\text { CC-0: } 81 \text { (58.3); CC- } \\
\text { 1: } 21 \text { (15.1); CC-2: } 30\end{array}$ & $\begin{array}{l}\text { Upfront: } 41 \\
\text { Interval: } 68\end{array}$ & $\begin{array}{l}1.7[33.3] \\
.9\end{array}$ & $\begin{array}{l}\text { Upfront: } 24 . \varepsilon \\
\text { Interval: } 16.8\end{array}$ & $\begin{array}{l}{[19.7]} \\
{[9.6]}\end{array}$ & - & - \\
\hline & & & cEOC & & & 12 & MMC & $\begin{array}{l}\text { Platinum-R: } \\
38.7\end{array}$ & $\begin{array}{l}(21.6) \\
\text { CC-3: } 7(5.0)\end{array}$ & $53.7[42.4]$ & & 29.6 [24.2] & & & \\
\hline & & & rEOC & & & 83 & & & & $23.5[18]$ & & $13.7[9.6]$ & & & \\
\hline
\end{tabular}




\begin{tabular}{|c|c|c|c|c|c|c|c|c|c|c|c|c|c|c|}
\hline $\begin{array}{l}\text { First authors } \\
\text { (ref.) }\end{array}$ & Year & $\begin{array}{l}\text { Case-control } \\
\text { or cohort }\end{array}$ & $\begin{array}{l}\text { Primitive/ } \\
\text { Recurrence }\end{array}$ & $\begin{array}{l}\text { FIGO (Inclusion of } \\
\text { persistent EOC } \text { S }^{\text {? }} \text { ) }\end{array}$ & $\begin{array}{l}\text { IP therapy } \\
\text { technique }\end{array}$ & no. of pts & Treatment and Chemotherapy regimen & $\begin{array}{l}\text { Platinum- } \\
\text { Sensitive pts }\end{array}$ & CC0-1 n (\%) & Median OS (mont) & hs) [5y-OS\%] & $\begin{array}{l}\text { Median DFS or PFS (months) } \\
\text { [5y-DFS\%] }\end{array}$ & $\begin{array}{l}\text { Morbidity (3-4 } \\
\text { grade/severe) } \%\end{array}$ & $\begin{array}{l}\text { Mortality } \\
\%\end{array}$ \\
\hline \multirow[t]{2}{*}{$\begin{array}{l}\text { Manzanedo } \\
(81)\end{array}$} & \multirow[t]{2}{*}{2017} & \multirow[t]{2}{*}{ Cohort } & $\mathrm{pEOC}$ & \multirow[t]{2}{*}{$\begin{array}{l}\text { IIIC-IV } \\
\text { (no) }\end{array}$} & \multirow[t]{2}{*}{ HIPEC } & $\begin{array}{l}31 \text { ( } 4 \text { up-front, } 27 \\
\text { inter-val) }\end{array}$ & \multirow{2}{*}{$\begin{array}{l}\text { NACT if not resectable pEOC, CT before } \\
\text { surgery in all rEOC + CRSSHIIPEC with } \\
\text { Taxol } 60 \mathrm{mg} / \mathrm{m}^{2} \text { or Cis } 100 \mathrm{mg} / \mathrm{m}^{2}+\text { Doxo } \\
15 \mathrm{mg} / \mathrm{m}^{2}\end{array}$} & - & \multirow[t]{2}{*}{$\begin{array}{l}\text { CC-0: } 56 \text { (92); CC-1: } \\
3 \text { (5); CC-2: } 2 \text { (3) }\end{array}$} & Not reached [55] & $\begin{array}{l}\text { Up-front CRS: [100]; } \\
\text { Interval CRS: } 40 \\
{[46.5]}\end{array}$ & 12 & \multirow[t]{2}{*}{$52.5(29.5)$} & \multirow[t]{2}{*}{0} \\
\hline & & & rEOC & & & 30 & & $\begin{array}{l}20 \text { Platinum-S, } \\
10 \text { Platinum-R }\end{array}$ & & $50[47,1]$ & $\begin{array}{l}\text { Platinum-S: } 59 \\
\text { [38.3]; Platinum-R: } \\
\text { Not reached [56] }\end{array}$ & 17 & & \\
\hline \multirow[t]{2}{*}{ Massari (82) } & \multirow[t]{2}{*}{2014} & \multirow[t]{2}{*}{ Cohort } & pEOC & \multirow[t]{2}{*}{$\begin{array}{l}\text { III-IV } \\
\text { (no) }\end{array}$} & \multirow[t]{2}{*}{ HIPEC } & $\begin{array}{l}14 \text { ( } 2 \text { up-front, } 12 \\
\text { inter-val) }\end{array}$ & \multirow{2}{*}{$\begin{array}{l}\text { CRS + HIPEC with Cis } 60 \mathrm{mg} / \mathrm{m}^{2} / \mathrm{L}+\text { Doxo } \\
20 \mathrm{mg} / \mathrm{m}^{2} / \mathrm{L} \text { or Taxotere } \\
60 \mathrm{mg} / \mathrm{m}^{2} / \mathrm{L} \text { + Doxo } 20 \mathrm{mg} / \mathrm{m}^{2} / \mathrm{L}\end{array}$} & \multirow[t]{2}{*}{-} & \multirow[t]{2}{*}{$\begin{array}{l}\text { CC-0: } 14 \text { (56); CC-1: } \\
8 \text { (32); CC-2,3: } 3(12)\end{array}$} & 36.5 & \multirow{2}{*}{$\begin{array}{l}\text { CC-0,1: 32.8; } \\
\text { CC2,3: 14; PCI 15: } \\
42 ; \mathrm{PCl}>15: 11\end{array}$} & 12.9 & \multirow[t]{2}{*}{$56(16)$} & \multirow[t]{2}{*}{8} \\
\hline & & & rEOC & & & 11 & & & & 27 & & 11.9 & & \\
\hline \multirow[t]{2}{*}{ Pavlov (83) } & \multirow[t]{2}{*}{2009} & \multirow[t]{2}{*}{ Cohort } & pEOC & \multirow[t]{2}{*}{ IIIC-IV } & \multirow[t]{2}{*}{ HIPEC+EPIC } & 31 & \multirow{2}{*}{$\begin{array}{l}\text { CRS+HIPEC with Doxo } 0.1 \mathrm{mg} / \mathrm{kg} / \mathrm{day}+ \\
\text { EPIC with Cis } 15 \mathrm{mg} / \mathrm{m}^{2} \text { for } 5 \text { days }\end{array}$} & \multirow[t]{2}{*}{-} & \multirow{2}{*}{$\begin{array}{l}\text { CC-0,1: } 52(92.8) \\
\text { CC-2,3: } 4(7.2)\end{array}$} & 34.1 & \multirow{2}{*}{$\begin{array}{l}\mathrm{PCl}>12:[38] ; \mathrm{PCl} \\
<12:[83]\end{array}$} & 26.2 & \multirow[t]{2}{*}{15} & \multirow[t]{2}{*}{3} \\
\hline & & & rEOC & & & 25 & & & & 40.1 & & & & \\
\hline \multirow[t]{2}{*}{ Pavlov (84) } & 2018 & Cohort & pEOC & - & HIPEC+EPIC & 55 & $\begin{array}{l}\text { CRS+EPIC with Doxo } 0.1 \mathrm{mg} / \mathrm{kg} \text { for } \\
2 \text { hours + Cis } 15 \mathrm{mg} / \mathrm{m}^{2} \text { for } 5 \text { days or }\end{array}$ & - & $\begin{array}{l}\text { CC-0,1: } 70(62.5) \\
\text { CC-2,3: } 1 \text { (25) }\end{array}$ & $40.3[24]$ & & - & $9.5(1.7)$ & 0.86 \\
\hline & & & rEOC & & & 61 & $\begin{array}{l}\text { HIPEC with Cis } 15 \mathrm{mg} / \mathrm{m}^{2}+\text { Doxo } \\
0.1 \mathrm{mg} / \mathrm{kg}\end{array}$ & $\begin{array}{l}\text { Platinum-R } \\
13.1 \% \\
\text { Platinum-S } \\
86.9 \%\end{array}$ & $\begin{array}{l}\text { CC-0,1: } 42(37.5) \\
\text { CC-2,3: } 3(75.0)\end{array}$ & 27.6 [3y-OS 16] & & & & \\
\hline Piso (85) & 2004 & Cohort & pEOC & IIIC-IV & HIPEC & 8 & (ev NACT) + CRS + HIPEC with Cis & - & CC-0: 9 (47.3) & 29 & & 18 & (36.8) & 5.7 \\
\hline & & & rEOC & & & 11 & $75 \mathrm{mg} / \mathrm{m}^{-2}$ or Mitoxantrone $15 \mathrm{mg} / \mathrm{m}^{-2}$ & & & 30 & & & & \\
\hline Robella (87) & 2014 & Cohort & $\begin{array}{l}\text { pEOC } \\
\text { rEO }\end{array}$ & III-IV (no) & HIPEC & $\begin{array}{l}45 \\
25\end{array}$ & $\begin{array}{l}\text { CRS + HIPEC with Cis } 100 \mathrm{mg} / \mathrm{m}^{2}+\text { Doxo } \\
15.2 \mathrm{mg} / \mathrm{L}\end{array}$ & - & $\begin{array}{l}\text { CC-0: } 55 \text { (78.6) } \\
\text { CC-1: } 9(12.8) \\
\text { CC-2: } 6 \text { (8.6) }\end{array}$ & $\begin{array}{l}48.0 \\
28.0\end{array}$ & 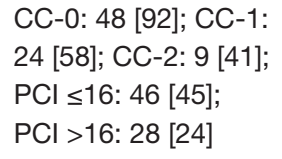 & - & $35.7(12.8)$ & 7.1 \\
\hline Roviello (88) & 2010 & Cohort & pEOC & IIIA-IIIC (yes) & HIPEC & 14 & $\begin{array}{l}\text { Primary CRS + post-op IV CT + } \\
\text { CRS +HIPEC with MMC } 25 \mathrm{mg} / \mathrm{m}^{2}+\text { Cis } \\
100 \mathrm{mg} / \mathrm{m}^{2}+\text { post-op IV CT }\end{array}$ & & $\begin{array}{l}\text { CC-0: } 11(79) \\
\text { CC-1: } 1 \text { (7) } \\
\text { CC-2,3: } 2 \text { (14) }\end{array}$ & [55] & $\begin{array}{l}\mathrm{PCI} 0:[100] ; \mathrm{PCl} \\
\text { 1-6: [48]; PCI>6 [0]; } \\
\text { CC-0: [71]; CC-1: }\end{array}$ & CC-0: [54] & $42(23)$ & 0 \\
\hline & & & & & & 31 & $\begin{array}{l}\mathrm{NACT}+\text { CRS + HIPEC with MMC } 25 \mathrm{mg} / \mathrm{m}^{2} \\
+ \text { Cis } 100 \mathrm{mg} / \mathrm{m}^{2}+\text { post-op IV CT }\end{array}$ & & $\begin{array}{l}\text { CC-0: } 20 \text { (64) } \\
\text { CC-1: } 4 \text { (13) } \\
\text { CC-2,3: } 7 \text { (23) }\end{array}$ & [58] & [44]; CC-2,3: [0] & & & \\
\hline & & & rEOC & & & 8 & $\begin{array}{l}\text { CRS+HIIPEC with MMC } 25 \mathrm{mg} / \mathrm{m}^{2}+\mathrm{Cis} \\
100 \mathrm{mg} / \mathrm{m}^{2}+\text { post-op IV CT }\end{array}$ & & $\begin{array}{l}\text { CC-0: } 6(75) \\
\text { CC-1: } 2(25)\end{array}$ & [44] & & & & \\
\hline Rufian (89) & 2006 & Cohort & pEOC & III & HIPEC & 19 & CRS+HIPEC with Paclitaxel $60 \mathrm{mg} / \mathrm{m}^{2}+$ & - & CC-0: 17 (52) & $38[37]$ & CC-0:[60] & 25 & (36) & 0 \\
\hline & & & rEOC & & & 14 & post-op IV & & CC-1,2: 16 (48) & 57 [51] & 1 & 31 & & \\
\hline $\begin{array}{l}\text { Sanchez- } \\
\text { Garcia (90) }\end{array}$ & 2016 & Cohort & $\mathrm{pEOC}$ & II-III-IV & $\begin{array}{l}\text { Modified } \\
\text { HIPEC }\end{array}$ & 16 & CRS+HIIPEC with Paclitaxel $175 \mathrm{mg} / \mathrm{m}^{2}$ & - & - & - & & - & $95.24(38.1)$ & 4.8 \\
\hline & & & rEOC & & & 5 & & & & & & & & \\
\hline
\end{tabular}




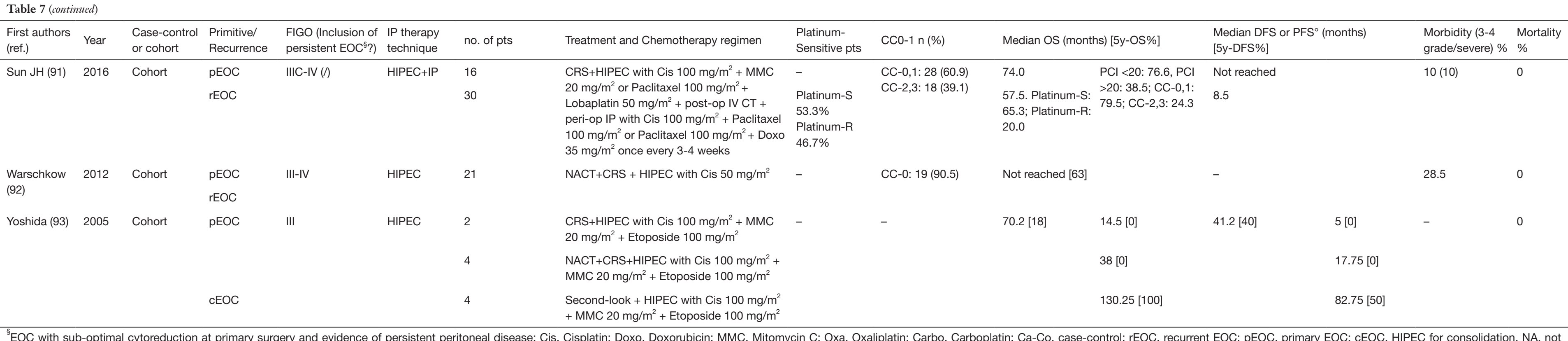

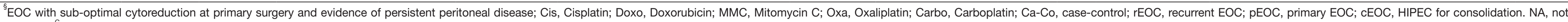

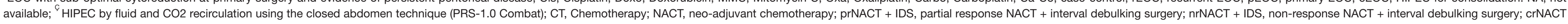
+ IDS, complete response NACT + interval debulking surgery; fr, first recurrence. 
Table 8 Prospective observational studies focus on intraperitoneal chemotherapy for primary and recurrent EOC

\begin{tabular}{|c|c|c|c|c|c|c|c|c|c|c|c|c|c|c|c|}
\hline \multirow{3}{*}{$\begin{array}{l}\begin{array}{l}\text { First authors } \\
\text { (ref.) }\end{array} \\
\text { Ansaloni (94 }\end{array}$} & \multirow{3}{*}{$\begin{array}{l}\text { Year } \\
2012\end{array}$} & \multirow{3}{*}{$\begin{array}{l}\begin{array}{l}\text { Case-control } \\
\text { or cohort }\end{array} \\
\text { Cohort }\end{array}$} & \multirow{2}{*}{$\begin{array}{l}\text { Primitive/ } \\
\text { Recurrence } \\
\text { pEOC }\end{array}$} & \multirow{3}{*}{$\begin{array}{l}\text { FIGO (Inclusion of } \\
\text { persistent EOC } \text { ? }) \\
\text { IIIC }\end{array}$} & \multirow{3}{*}{$\begin{array}{l}\text { IP therapy } \\
\text { technique } \\
\text { HIPEC }\end{array}$} & \multirow{3}{*}{$\begin{array}{l}\text { no. of pts } \\
9 \\
30\end{array}$} & \multirow{3}{*}{$\begin{array}{l}\text { Chemotherapy regimen } \\
\text { CRS+HIPEC with Cis } 100 \mathrm{mg} / \mathrm{m}^{2} \text { and } / \mathrm{or} \\
\text { Paclitaxel } 175 \mathrm{mg} / \mathrm{m}^{2} \text { and } / \mathrm{or} \mathrm{Doxo} 35 \mathrm{mg} / \mathrm{m}^{2}\end{array}$} & \multirow{3}{*}{$\begin{array}{l}\text { Platinum-Sensitive } \\
\text { pts } \\
\text { Platinum-S: } 33 \%, \\
\text { Platinum-R: } 62 \%\end{array}$} & \multirow{3}{*}{$\begin{array}{l}\text { CC0-1 n (\%) } \\
\text { CC-0: } 35 \text { (90), CC-1: } 3 \\
(7), \text { CC-3: } 1 \text { (3) }\end{array}$} & \multicolumn{2}{|c|}{ OS (months) [5y-OS\%] } & \multicolumn{2}{|c|}{ DFS or PFS (months) } & \multirow{3}{*}{$\begin{array}{l}\text { Morbidity (3-4 } \\
\text { grade/severe) \% } \\
(18)\end{array}$} & \multirow{3}{*}{$\begin{array}{l}\text { Mortality \% } \\
2.5\end{array}$} \\
\hline & & & & & & & & & & - & & Mean & DFS: 14.4 & & \\
\hline & & & rEOC & & & & & & & & & & & & \\
\hline \multirow[t]{2}{*}{ Ansaloni (95) } & \multirow[t]{2}{*}{2015} & \multirow[t]{2}{*}{ Cohort } & pEOC & \multirow[t]{2}{*}{ IIIC-IV } & \multirow[t]{2}{*}{ HIPEC } & 9 & \multirow{2}{*}{$\begin{array}{l}\text { CRS }+ \text { HIPEC with Cis } 100 \mathrm{mg} / \mathrm{m}^{2} \text { and Paclitaxel } \\
175 \mathrm{mg} / \mathrm{m}^{2}\end{array}$} & \multirow[t]{2}{*}{ Platinum-S: 100\% } & \multirow[t]{2}{*}{ CC-0: $13(100)$} & \multirow{2}{*}{\multicolumn{2}{|c|}{-}} & \multirow{2}{*}{\multicolumn{2}{|c|}{-}} & \multirow[t]{2}{*}{$100(61.5)$} & \multirow[t]{2}{*}{0} \\
\hline & & & rEOC & & & 4 & & & & & & & & & \\
\hline \multirow{2}{*}{$\begin{array}{l}\text { Coccolini } \\
\text { (96) }\end{array}$} & \multirow[t]{2}{*}{2015} & \multirow[t]{2}{*}{ Cohort } & pEOC & \multirow[t]{2}{*}{ IIIC-IV } & \multirow[t]{2}{*}{ HIPEC } & 30 & \multirow{2}{*}{$\begin{array}{l}\text { (ev. NACT) + CRS+HIPEC with Cis } 100 \mathrm{mg} / \mathrm{m}^{2} \\
+ \text { Paclitaxel } 175 \mathrm{mg} / \mathrm{m}^{2}\end{array}$} & \multirow{2}{*}{$\begin{array}{l}\text { Platinum-S: } 68.5 \% \text {, } \\
\text { Platinum-R: } 31.49 \%\end{array}$} & \multirow{2}{*}{$\begin{array}{l}\text { CC-0: } 47 \text { (87), CC-1: } 7 \\
(13)\end{array}$} & 32.9 & 22 & 12.5 & 13 & $95.2(35.2)$ & 5.6 \\
\hline & & & rEOC & & & 24 & & & & & 44 & & 12 & & \\
\hline Di Giorgio & 2008 & Cohort & pEOC & IIIC-IV & HIPEC & 22 & CRS+HIPEC with Cis $75 \mathrm{mg} / \mathrm{m}^{2}$ & - & CC-0,1: 41 (87.2), CC- & 27 & $\mathrm{PCl}<15: 24 ; \mathrm{PCl}>15: 26$ & 25.5 & $\mathrm{PCl}<15: 21 ; \mathrm{PCl}>15: 20$ & $48.9(21.3)$ & 4.2 \\
\hline (79) & & & rEOC & & & 25 & & & 2,3: $6(12.8)$ & 22.5 & $\begin{array}{l}\text { CC-0: 26; CC-1: 13; CC- } \\
\text { 2,3: } 12\end{array}$ & 15.5 & $\begin{array}{l}\text { CC-0: 24; CC-1: 13; CC- } \\
2,3: 6\end{array}$ & & \\
\hline Gonzalez & 2013 & Cohort & pEOC & IIIC-IV & HIPEC + & 15 & $\mathrm{NACT}+\mathrm{CRS}+\mathrm{HIPEC}$ with Cis $100 \mathrm{mg} / \mathrm{m}^{2}+$ & - & CC-0: $11(73.3)$ & 77.8 & & $21.1(7$ & & 26.6 & 6.6 \\
\hline Bayon (97) & & & rEOC & & & $\begin{array}{l}19 \text { first } \\
\text { recurrence, }\end{array}$ & $\begin{array}{l}\text { Doxo } 30 \mathrm{mg} / \mathrm{m}^{2}+\text { ev. EPIC with Taxol } 20 \mathrm{mg} / \mathrm{m}^{2} \\
\text { for } 5 \text { days }\end{array}$ & & $\begin{array}{l}\text { First recur. CC-0: } 14 \\
\text { (73.6) }\end{array}$ & First $r$ & cur. 62.8 & First $\mathrm{re}$ & cur. $18.1(62.2)$ & 21 & 5.2 \\
\hline & & & & & & $\begin{array}{l}8 \text { second } \\
\text { recurrence }\end{array}$ & & & $\begin{array}{l}\text { Second or subsequent } \\
\text { recurrence CC-0: } 6 \text { (75) }\end{array}$ & $\begin{array}{l}\text { Secon } \\
\text { recurr }\end{array}$ & $\begin{array}{l}\text { d or subsequent } \\
\text { nce: } 35.7\end{array}$ & $\begin{array}{l}\text { Secon } \\
\text { recurre }\end{array}$ & $\begin{array}{l}\text { d or subsequent } \\
\text { ence: } 5.7(17.9)\end{array}$ & 37.5 & 12.5 \\
\hline Pomel (98) & 2010 & Cohort & $c E O C+r E O C$ & IIIC (yes) & HIPEC & 31 & $\begin{array}{l}\text { CRS + post-op IV CT with Cis } 6 \text { cycles + } \\
\text { second-look laparotomy + HIPEC with Oxa } \\
460 \mathrm{mg} / \mathrm{m}^{2}\end{array}$ & - & CC-0 $31(100)$ & {$[2 y-0 \leq$} & 67\%] & {$[2 y-D F$} & s 27\%] & (29) & 0 \\
\hline Tentes (99) & 2012 & Cohort & PEOC & - & HIPEC & 23 & CRS+HIPEC with Cis $50 \mathrm{mg} / \mathrm{m}^{2}+$ Doxo & - & CC-0: $30(69.8)$ & 37 & & - & & (14) & 4.7 \\
\hline & & & rEOC & & & 20 & $10 \mathrm{mg} / \mathrm{m}^{2}$ in Platinum- $\mathrm{R}$ & & & & & & & & \\
\hline
\end{tabular}

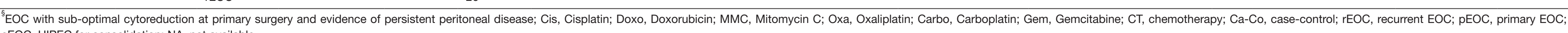
SOCC, HIPEC for consolidation: NA, not available. 
CRS+HIPEC or with systemic chemotherapy alone. The significant benefit in median DFS that was observed in the HIPEC group remained regardless of the patients' BRCA status (BRCAwt: 21.8 in HIPEC group vs. 12.1 in control group, $\mathrm{P}=0.011$; BRCAmut: 20.9 in HIPEC group $v s .12 .6$ in control group, $\mathrm{P}=0.012$ ). The observed benefit in OS remained significant in BRCAmut patients treated with HIPEC and showed a trend toward a benefit in BRCAwt patients (BRCAwt: 61.6 in HIPEC group vs. 47.7 in control group, $\mathrm{P}=0.068$; BRCAmut: 80.1 in HIPEC group vs. 71.6 in control group, $\mathrm{P}=0.036$ ).

In eight case-control studies $(35,47,55-57,60,62,77)$, almost all focused on platinum-S patients. They compared outcomes of patients treated with CRS + HIPEC with patients treated with CRS alone for rEOC. Most of the studies $(47,55,57,62)$ showed a significant benefit in terms of OS and DFS in patients treated with HIPEC. Other studies showed better outcomes in HIPEC group but without statistical significance $(38,56,60)$.

There is only one RCT that included 120 patients with rEOC (FIGO IIIC-IV) comparing CRS + HIPEC + systemic chemotherapy with CRS + systemic chemotherapy. The study showed a significant higher OS in the HIPEC group (26.9 vs. 14.2 months in stage IIIC and 26.4 vs. 11.9 months in stage IV, $\mathrm{P}=0.006)$. Furthermore, in the HIPEC group similar OS in platinum-S and platinum-R patients was observed while in the control group the OS of platinum-S patients was significantly longer than platinum- $\mathrm{R}$ ones (15.2 vs. 10.2, $\mathrm{P}=0.002)$. Median $\mathrm{OS}$ in HIPEC group was significantly higher than in CRS group both in $\mathrm{PCI}<15(\mathrm{P}=0.031)$ and in $\mathrm{PCI}>15$ patients $(\mathrm{P}=0.049)$. This benefit remained only in CC-0 patients (30.9 vs. 16.9 months, $\mathrm{P}=0.038)$.

The study by Spiliotis (Table 1) was criticized by some authors (100) regarding methods and statistical analysis. The endpoints, the randomization procedure and the systemic CT regimen were not explained and DFS, morbidity and mortality were not reported. In two recent meta-analyses $(101,102$ ) including observational studies and the only RCT on rEOC, CRS + HIPEC showed a significant advantage compared to CRS alone in terms of OS and DFS (if CC-3 patients were excluded). Almost all the studies agree in affirming the completeness of the cytoreduction (CC- 0 ) as the major prognostic factor on OS and DFS $(18,34,39,42,44,52,57,69,76)$. The reported major morbidity and mortality for CRS + HIPEC in patients with $\mathrm{rEOC}$ ranges from 8.3 to $72 \%$ and from 0 to $22.2 \%$ respectively. Then, evidence to date suggests a role for
HIPEC in both platinum-R and platinum-S patients and in both BRCAmut and BRCAwt patients with rEOC, but further phase III trial are needed in this setting.

\section{Other intraperitoneal chemotherapy}

A large case-control propensity-score study by Lu et al. (64) on 310 patients with rEOC compared CRS associated with platinum-based NIPEC with CRS associated with IV CT, showing significantly longer DFS in the NIPEC group, both in platinum-S and platinum-R patients (4.9 vs. 2.4 months, $\mathrm{P}<0.001$, for platinum- $\mathrm{R}$ disease, and 9.8 vs. 6.9 months, $\mathrm{P}<0.001$, for platinum-S disease). Three prospective observational studies (71-73) focusing on $\mathrm{rEOC}$ treated with PIPAC with cisplatin and doxorubicin showed a histologic tumor regression in 64\% with median OS ranging from 13.6 to 14.7 months. The reported OS and DFS obtained with IP CT other than HIPEC in rEOC ranged from 13.6 to 25.5 months and from 2.4 to 9.8 months, respectively.

\section{Consolidation intraperitoneal chemotherapy}

Intraperitoneal chemotherapy has also been applied as consolidation treatment when patients present complete response to primary treatment (CRS and systemic chemotherapy) to reduce the chance of recurrence.

\section{HIPEC}

Several non-randomized reports (Tables 9,10) have investigated the use of second-look surgery with HIPEC as additional treatment following a complete response to frontline therapy. The median OS and DFS of patients with EOC treated with consolidation HIPEC ranges from 14 to 64.4 moths (5y-OS 70-84.21\%) and from 13 to 18.5 months (5y-DFS 45-63\%) respectively. Some case-control studies (105-107) compared consolidation HIPEC after CRS and post-operative systemic chemotherapy with no further therapies. In the study by Gori et al. (105) patients in the HIPEC group reached a median OS of 64.4 months (5y-OS $70 \%)$ and a $5 y-D F S$ of $45 \%$ compared with 46.4 months (5y-OS $58 \%$ ) and $0 \%$ in the control group. In the study by Kim et al. (106) patients in the HIPEC group showed significantly higher $8 \mathrm{y}-\mathrm{OS}(84 \%$ vs. $25 \%, \mathrm{P}=0.0004)$ and $8 \mathrm{y}$-DFS $(63 \%$ vs. $29 \%, \mathrm{P}=0.027)$.

A relative advantage of HIPEC delivery at the time of consolidation is the potential for a reduction in the toxicity of associated CRS (29). The major morbidity associated 

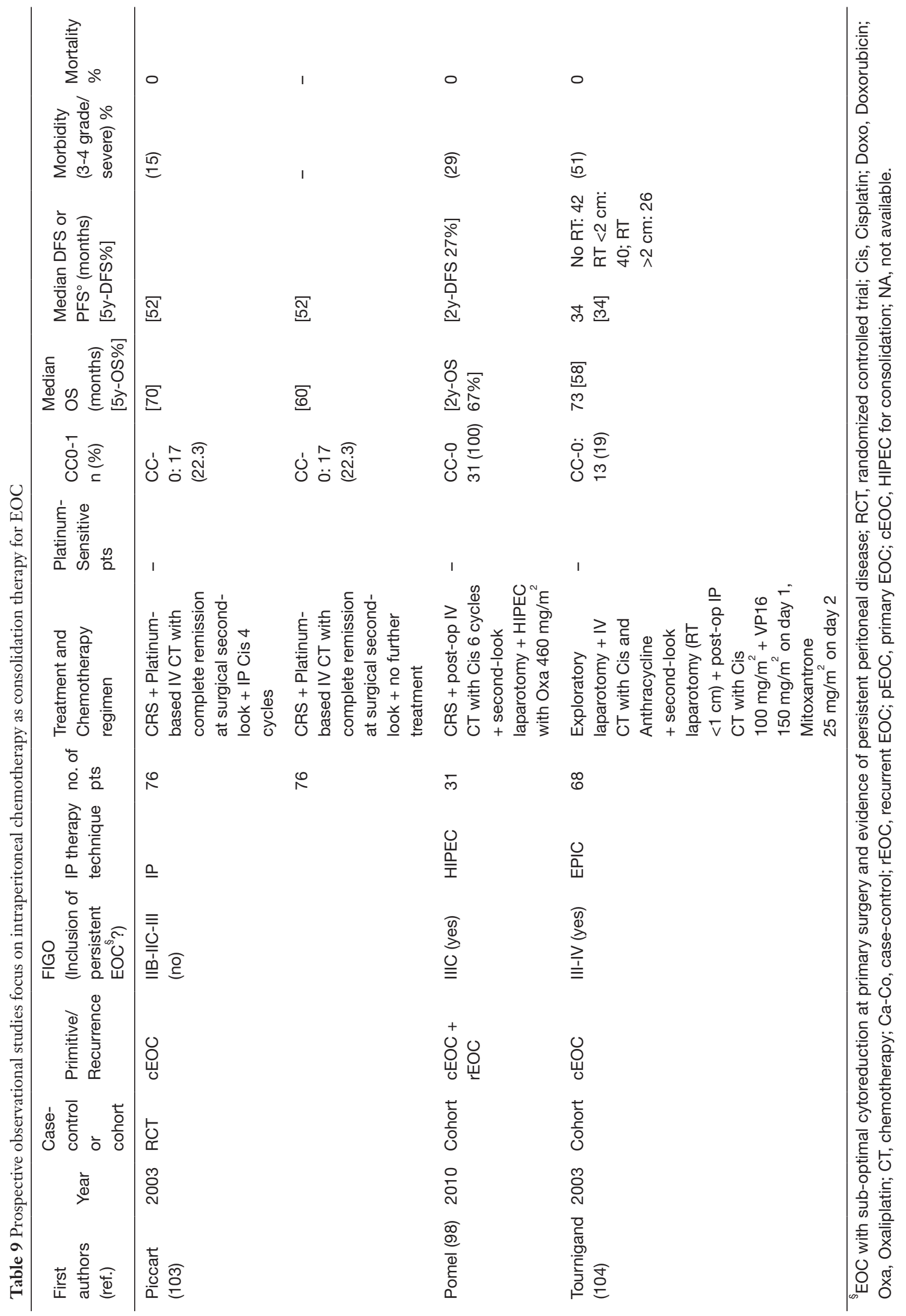

(C) Journal of Gastrointestinal Oncology. All rights reserved. 


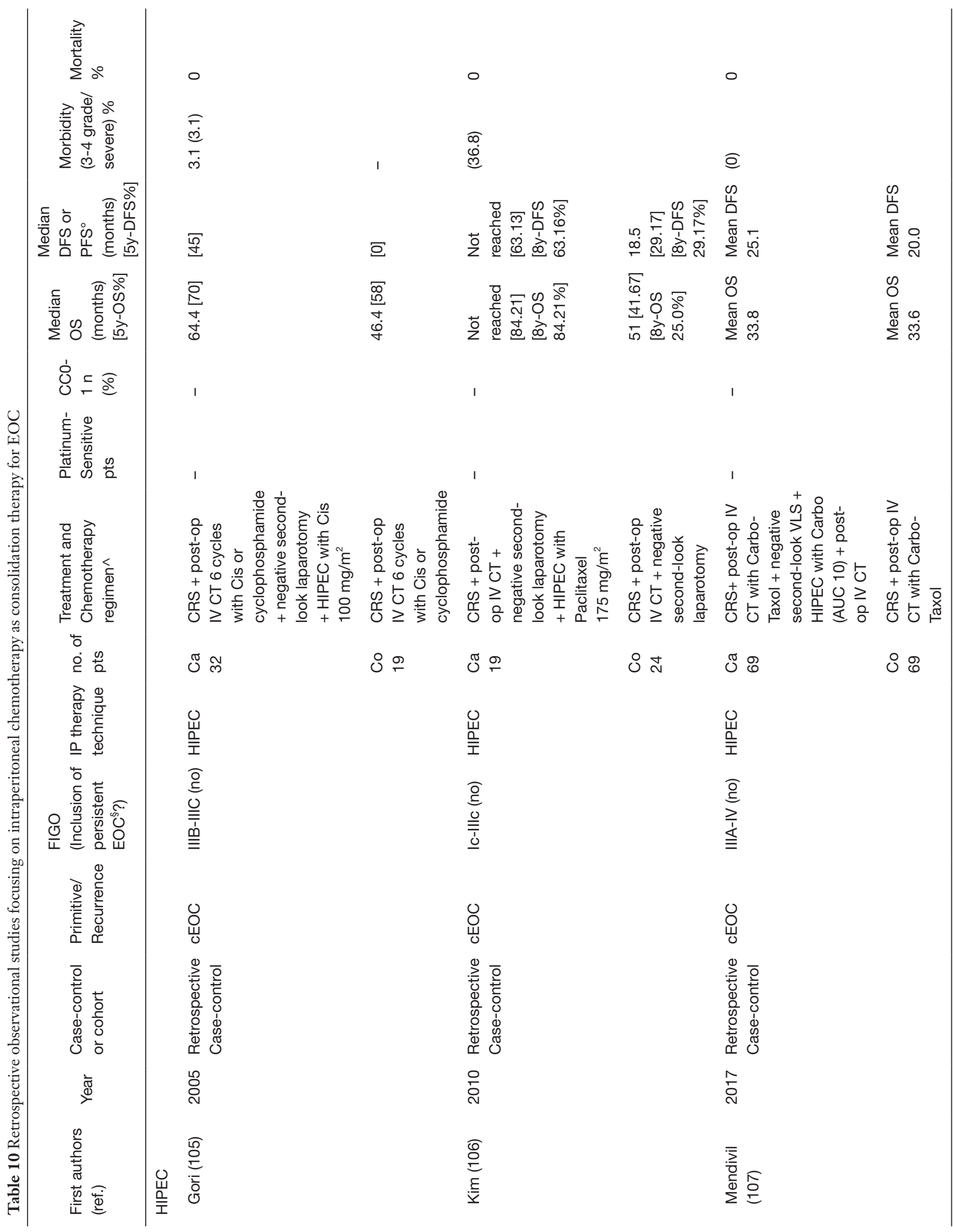



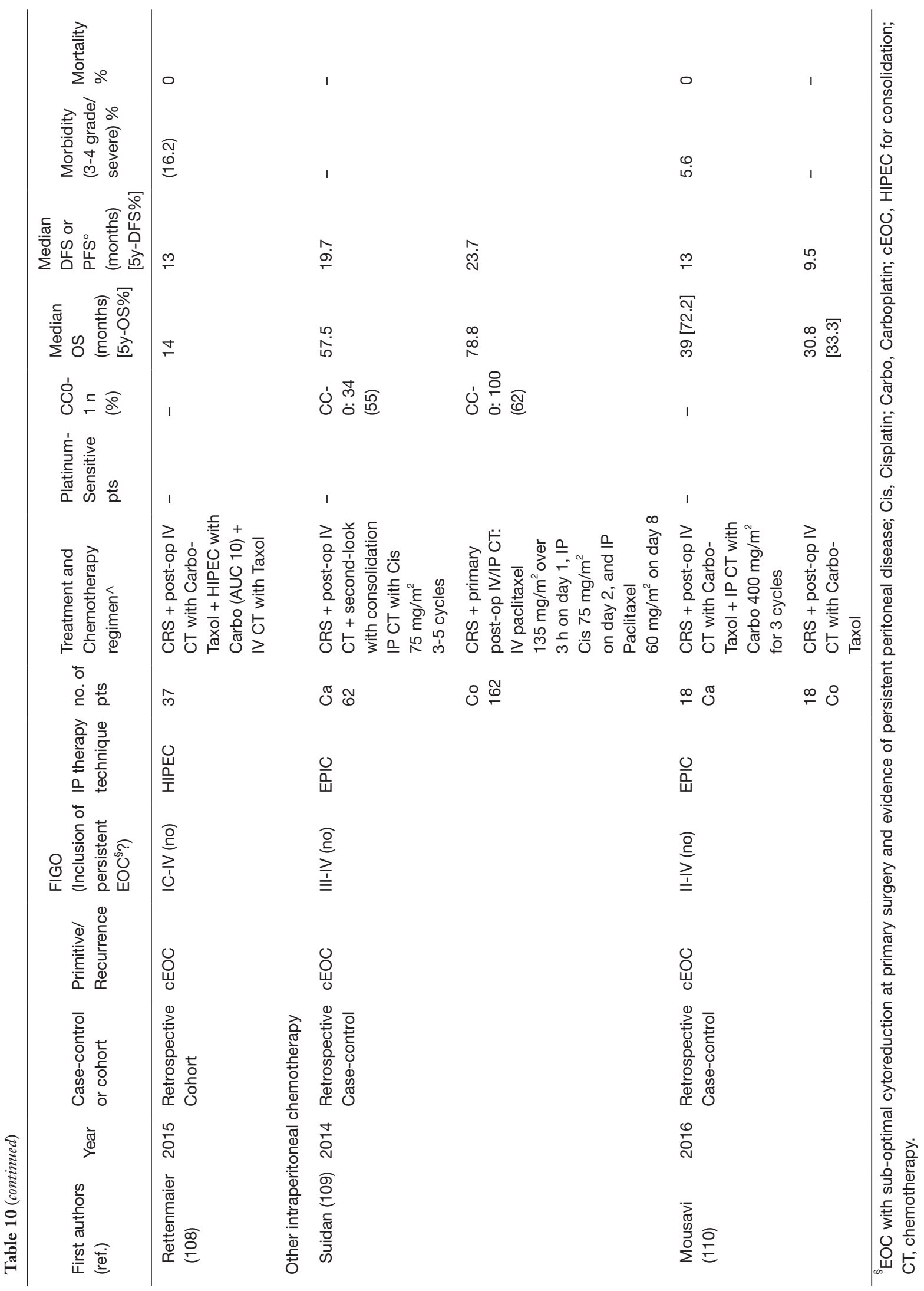
with consolidation HIPEC ranges from $0 \%$ to $51 \%$ and the mortality is $0 \%$ in all studies.

\section{Other intraperitoneal chemotherapy}

The RCT by Piccart on 152 patients with stage IIB-IICIII EOC treated with CRS and platinum-based systemic CT with evidence of complete remission at surgical secondlook, compared patients treated with post-operative consolidation NIPEC cisplatin (administered through an intraperitoneal catheter) with Cisplatin with patients treated with no further therapies. In the NIPEC group, the $5 \mathrm{y}$-OS and $5 \mathrm{y}-\mathrm{DFS}$ were $70 \%$ and $53 \%$ respectively, compared to $60 \%$ and $52 \%$ in control group. The respective hazard ratios for DFS and OS with 95\% CI: were 0.89 (0.59$1.33)$ and 0.82 (0.52-1.29). For the NIPEC consolidation CT (other than HIPEC) median OS ranges from 39 to 73 months (5y-OS 58-72\%) and median DFS from 13 to 34 months (5y-DFS $34-52 \%$ ). Suidan et al. compared survival outcomes for patients with advanced EOC who received primary systemic and IP chemotherapy to those who received systemic CT followed by consolidation IP chemotherapy. In this study primary IP chemotherapy was associated with improved OS and with the same DFS compared to systemic CT followed by consolidation IP $\mathrm{CT}$ in patients with optimally cytoreduced advanced EOC (median OS 78.8 vs. 57.5 months, $\mathrm{P}=0.004$; median DFS 23.7 vs. 19.7 months, $\mathrm{P}=0.11$ ).

\section{Early postoperative intraperitoneal chemotherapy (EPIC)}

Early postoperative intraperitoneal chemotherapy (EPIC) presents some potential advantages in respect to the HIPEC $(84,111)$. In fact, it is administered immediately after the CRS and inside the abdomen when the tumor burden within the abdominal cavity is minimal. EPIC timing and way of administration may allow an effective penetration within sites of wound healing potentially reducing the possibility to have cancer cells entrapped within fibrin deposits and scars.

EPIC associated potential disadvantages are the increased rate of postoperative morbidity and infections (112-114). EPIC does not necessitate hyperthermia and may be utilized after HIPEC or CRS alone. It is usually administered within the $4^{\text {th }}-5^{\text {th }}$ post-operative day through the abdominal drains placed during surgery. Therapy cycles usually last for 24 hours ensuring an adequate exposure of the tumor cells to the drugs. Suggested drugs for EPIC are the cell-cycle specific such as 5-fluorouracil and taxanes $(115,116)$.

An alternative to EPIC is the dose-dense early postoperative intraperitoneal chemotherapy (DD-EPIC) given in front-line. It seems to give good results. In fact, some data showed that DD-EPIC seems to significantly increase non-progression rate in advanced OC. A phase 2 trial where 218 patients with FIGO IIIC-IV OC were randomly allocated to receive DD-EPIC followed by intravenous (IV) chemotherapy (DD-EPIC group), or IV chemotherapy alone (IV group) reported a median OS of 67.5 and 46.3 months in the DD-EPIC and IV group, respectively. Estimated OS at 5 years was $61.0 \%$ with DD-EPIC, and $38.2 \%$ with IV. Estimated PFS at 5 years was $26.0 \%$ vs. $8.5 \%$ for DD-EPIC and intravenous, respectively (117).

\section{Intraperitoneal drugs in ovarian cancer:}

Several drugs have been utilized intraperitoneally in treating EOC. Dosages, perfusion times, and methodologies are different across the different centers. Even if supported by definitive scientific literature, most of these drugs have never been recognized as officially applicable within the peritoneal cavity. In several cases the use of IP drugs for HIPEC, EPIC or NIPEC administration is off-label under the direct responsibility of the oncologists and surgeons.

Cisplatin (cis-diamminedichloroplatinum-III, CDDP) action works through the formation of adducts to DNA causing cells apoptosis (118). CDDP can be applied in normothermia or in hyperthermia. Hyperthermia seems to augment the CDDP effect (119-121). The main concern in CDDP use is the potential nephrotoxicity (122). However, it has been questioned if the potential nephrotoxicity is mainly due to the renal excretion and the consequent potential toxicity, or to the fact that surgical physiological load of extensive CRS on already unhealthy kidneys may promote a secondary-hit related renal injury leading to renal insufficiency (123). This may necessitate, in the most severe cases, transient or definitive renal replacement therapy. The toxicity, in fact, seems to be related to the aggressive CRS and not only to drug exposure (95). Renal failure is generally relatively low and under the toxicity threshold (95). Moreover, the broad heterogeneity in the CDDP dosages throughout the different trials is not correlated to the different complication rates, further suggesting the correlation of the complications rate to the CRS procedure 
and not only to the CDDP administration.

Taxanes act by stabilizing the microtubule against depolymerization thereby disrupting normal microtubule dynamics and preventing the cells to perform their normal activity in a cell cycle-specific way (124). The main characteristic of paclitaxel and docetaxel is the high molecular weight that allows for a high area under the curve (AUC) ratio of 853 and 861, respectively (125). This characteristic contributes to give to these drugs a clear pharmacokinetic advantage for IP administration (126). Conflicting results exist about the possible thermal augmentation of this class of drugs (126). Taxanes have been used in a neoadjuvant intraperitoneal setting as well as EPIC or adjuvant post-operative repetitive administrations. The research interest is to increase their bioavailability.

Doxorubicin or hydroxyldaunorubicin (adriamycin) is part of the antibiotic family of chemotherapy agents and precisely an anthracycline. It acts depending on the temperature modifying the cell membrane $(127,128)$. Doxorubicin has a favorable AUC ratio of 230 due to the high molecular weight (129-133). The toxicity encountered in intravenous administration is a dose limiting cardiotoxicity that is not present with IP delivery. A mild thermal augmentation has been demonstrated for doxorubicin (134). PEGylated liposomal doxorubicin seems to have even more favorable pharmacokinetic effect (135).

Mitomycin $C$ acts by cross-linking DNA with the antibiotic type molecule. It needs to be activated to enter the cells and be effective (136). Its AUC is 23.5 and this quality associated with thermal enhancement give the molecule a favorable action in HIPEC administration (137).

Oxaliplatin (oxalato-1,2-diaminocyclohexane-platinum (II)) has a very low AUC and a rapid absorption into the tissues. For these reasons oxaliplatin is usually administered during HIPEC with short application times. Hyperthermia enhance its effect on tumor cells but oxaliplatin-based HIPEC increase the risk of bleedings $(119,138)$. In general, oxaliplatin should be infused within a dextrose-based carrier because of instability in other solutions (139).

Convincing data exist concerning the synergism between heat and the activity of many antineoplastic drugs against tumor cells growing in vitro $(91,119)$. Pharmacokinetic data provide a credible rationale for HIPEC. However more data about the different pharmacological aspects and comparative efficacy studies between the different drugs are needed $(98,112,140)$. The pharmacokinetics of several antineoplastic drugs utilized during HIPEC have been defined $(61,116)$ especially for cisplatin $(63)$ and paclitaxel, which are among the most effective against EOC. Good results have been demonstrated with the administration of the two drugs together during HIPEC $(28,95,96)$. A comparable concentration of cisplatin + paclitaxel in the peritoneal tissue and in the perfusate during HIPEC have been demonstrated, showing a good antineoplastic effect with low systemic drug absorption. This will give the maximal anticancer effect with low risk of side-effects due to systemic drug circulation (95). However, the pharmacokinetics of these molecules was investigated during and after intraperitoneal administration with hyperthermia only when infused alone and not in combination. It is not possible to exclude pharmacokinetic interaction between these two different drugs. Some studies are trying to compare the effect of the different molecules when administered intraperitoneally with hyperthermia.

A prospective cohort of 41 patients with stage IIIC or IV EOC treated with CRS and HIPEC, where analyzed according to the two combinations of drugs. Cisplatin/ doxorubicin were given to 19 patients $(46 \%)$ and paclitaxel to the other 22 patients (54\%). No difference in morbidity and mortality rate and survival rates were demonstrated within the two groups. The $3 y-O S$ was $66 \%$ in cisplatin + doxorubicin group and $82.9 \%$ in paclitaxel group $(\mathrm{P}=0.248)(141)$.

\section{Results}

Present study included 57 patients: 35 with pEOC and 22 with rEOC. Three of the 35 patients with pEOC were treated with upfront CRS+HIPEC, while 32 with interval CRS+HIPEC. Pre-operative and intra-operative data are showed in Table 11. Mean PCI was $11.93 \pm 9.18$. In the $89.5 \%$ of patients CC- 0 was obtained. The $84.2 \%$ of patients received Cisplatin + Taxol as HIPEC regimen. Major complication rate and mortality rate were $35.1 \%$ and $1.8 \%$ respectively. Re-operation rate was $12.3 \%$. The mean ICU length of stay (LOS) was 4.25 days (SD 9.7, median 2, range $0-54)$. The mean total LOS was 27.18 days (SD 24.00, median 20, range 10-124). The $70.2 \%$ of patients received post-operative IV CT. The mean OS for pEOC was 40.2 months (SE 3.9, 95\% CI: 32.5-47.9). The median OS for pEOC was not reached. The median DFS for pEOC was 13 months (SE 1.7, 95\% CI: 9.7-16.3), with $2 y-O S$ of $71 \%$ and $2 y-D F S$ of $37 \%$. The median OS and DFS for rEOC were 46 months (SE 0.0) and 11 months (SE $2.9,95 \%$ CI: $5.2-16.7)$ respectively, with $2 y-O S$ of $68 \%$ and $2 \mathrm{y}$-DFS of $34 \%$. There was no significant difference in OS 
Table 11 Patients' characteristics

\begin{tabular}{|c|c|}
\hline Characteristics & $\begin{array}{c}\mathrm{n}(\%) \text { or mean } \pm \\
\mathrm{SD} / \text { median (min, max) }\end{array}$ \\
\hline Primary EOC & $35(61.4)$ \\
\hline Upfront CRS + HIPEC & $3(5.3)$ \\
\hline Interval CRS + HIPEC & $32(56.1)$ \\
\hline Recurrent EOC & $22(38.6)$ \\
\hline Platinum-sensitive & 7 (12.3) \\
\hline Missing & $15(26.3)$ \\
\hline \multicolumn{2}{|l|}{ FIGO stage } \\
\hline IIIC & $46(80.7)$ \\
\hline IV & $10(17.5)$ \\
\hline Missing & $1(1.8)$ \\
\hline \multicolumn{2}{|l|}{ Grading } \\
\hline G1 & $2(3.5)$ \\
\hline G2 & $5(8.8)$ \\
\hline G3 & $44(77.2)$ \\
\hline Missing & $6(10.5)$ \\
\hline \multicolumn{2}{|l|}{ BRCA status } \\
\hline BRCA wild type & $11(19.3)$ \\
\hline Missing & $46(80.7)$ \\
\hline \multicolumn{2}{|l|}{ Histology } \\
\hline $\begin{array}{l}\text { Epithelial adenocarcinoma } \\
\text { NOS }\end{array}$ & $5(8.8)$ \\
\hline Serous adenocarcinoma & $44(77.1)$ \\
\hline Clear-cell adenocarcinoma & $1(1.8)$ \\
\hline Endometrioid carcinoma & $6(10.5)$ \\
\hline Mucinous adenocarcinoma & $1(1.8)$ \\
\hline \multicolumn{2}{|l|}{$\mathrm{PCl}$} \\
\hline $\mathrm{PCl}<15$ & $36(63.2)$ \\
\hline $\mathrm{PCl} \geq 15$ & $20(35.1)$ \\
\hline Missing & $1(1.8)$ \\
\hline \multicolumn{2}{|l|}{$\mathrm{CC}$} \\
\hline CC-O & $51(89.5)$ \\
\hline CC-1 and CC-2 & $6(10.5)$ \\
\hline
\end{tabular}

Table 11 (continued)
Table 11 (continued)

\begin{tabular}{|c|c|}
\hline Characteristics & $\begin{array}{c}\mathrm{n}(\%) \text { or mean } \pm \\
\mathrm{SD} / \text { median }(\min , \max )\end{array}$ \\
\hline \multicolumn{2}{|l|}{ HIPEC regimen } \\
\hline Cisplatin + Taxol & $48(84.2)$ \\
\hline Cisplatin + Mitomycin & $2(3.5)$ \\
\hline Cisplatin + Doxorubicin & $5(8.8)$ \\
\hline Cisplatin alone & $2(3.5)$ \\
\hline \multicolumn{2}{|c|}{ Pre-HIPEC chemotherapy regimen } \\
\hline \multicolumn{2}{|l|}{ Primary EOC (n=35) } \\
\hline NACT & $30(85.7)$ \\
\hline Carboplatin + Taxol & $2(5.7)$ \\
\hline \multicolumn{2}{|l|}{$\begin{array}{l}\text { Carboplatin }+ \text { Taxol }+ \\
\text { Bevacizumab }\end{array}$} \\
\hline No NACT & $3(8.6)$ \\
\hline \multicolumn{2}{|c|}{$\begin{array}{l}\text { In recurrent EOC }(\mathrm{n}=22) \text { (more than one regimen for each } \\
\text { patient) }\end{array}$} \\
\hline Carboplatin + Taxol & $16(72.7)$ \\
\hline Bevacizumab & $5(22.7)$ \\
\hline Niraparib & $1(4.5)$ \\
\hline Doxorubicin & $6(27.3)$ \\
\hline Trabectedin & $3(13.6)$ \\
\hline Gemcitabine & $2(9.0)$ \\
\hline Etoposide & $1(4.5)$ \\
\hline Age & $58.33 \pm 8.64 / 59(42-73)$ \\
\hline $\mathrm{PCl}$ & $11.93 \pm 9.18 / 12(0-37)($ IQR 15) \\
\hline
\end{tabular}

and DFS between pEOC and rEOC (Figure 2).

No significant difference in OS and DFS was found between patients with FIGO stage IIIC and IV.

Among patients with rEOC, CC-0 patients had significantly longer median OS than CC-1,2 patients (46 vs. 4 months, $\mathrm{P}<0.001$ ) with $2 \mathrm{y}-\mathrm{OS}$ of $76 \%$ vs. $0 \%$. Furthermore, patients with rEOC and PCI $<15$ had significantly longer median OS than patients with $\mathrm{PCI}>14$ (46 vs. 19 months, $\mathrm{P}=0.014$ ) with $2 \mathrm{y}$-OS of $100 \%$ vs. $29 \%$. Tables 12 reports univariate and multivariate analysis of factors influencing OS. Cisplatin + Taxol as IP CT regimen 

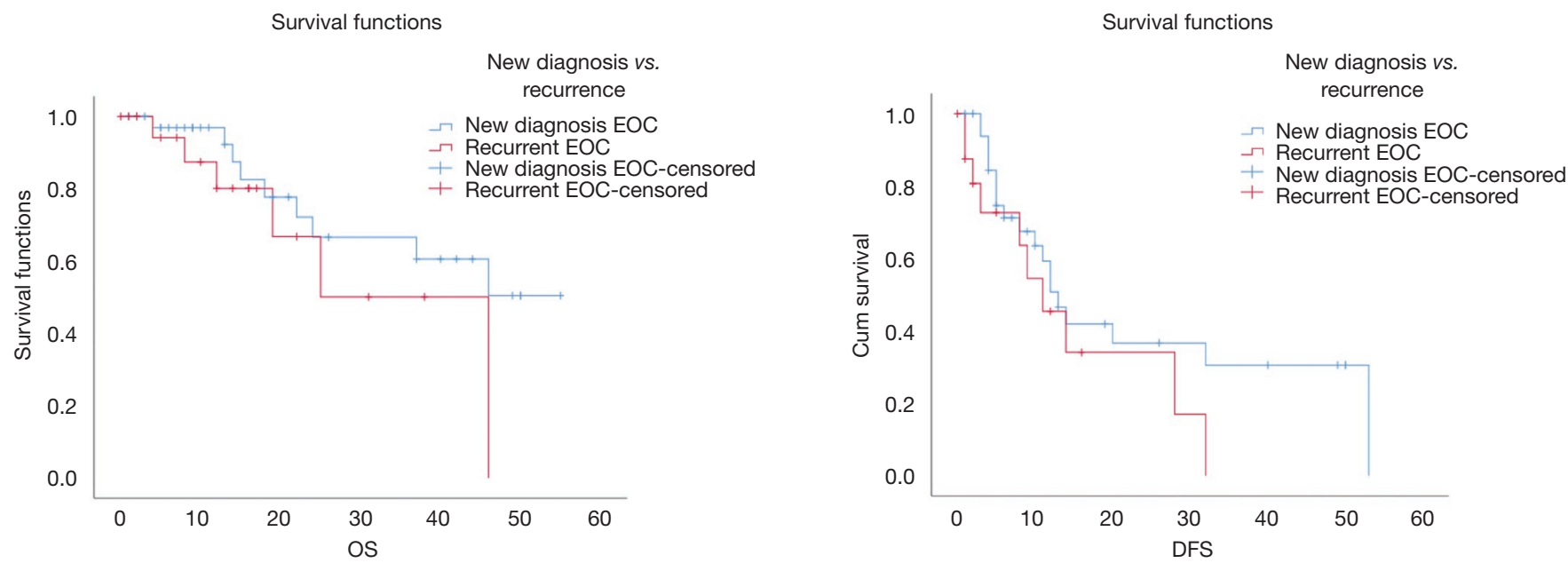

Figure 2 Kaplan-Meier survival curves of primary and recurrent epithelial ovarian cancer.

Funzioni di sopravvivenza

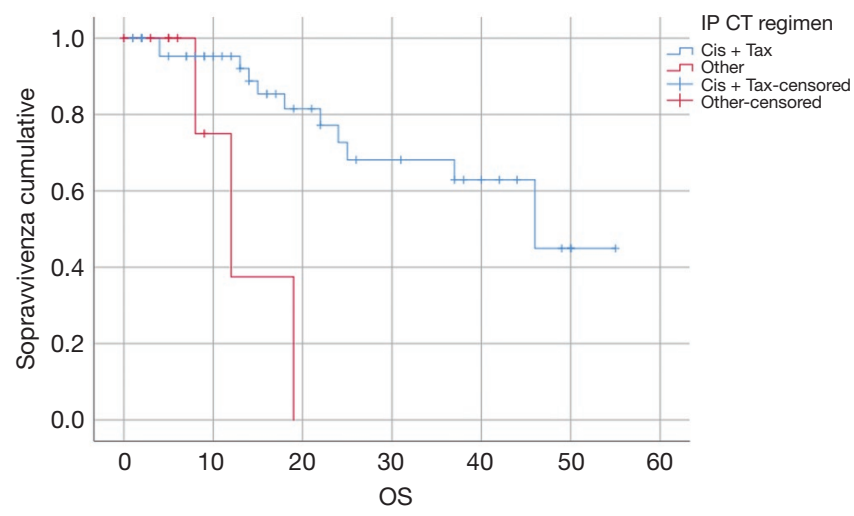

Funzioni di sopravvivenza

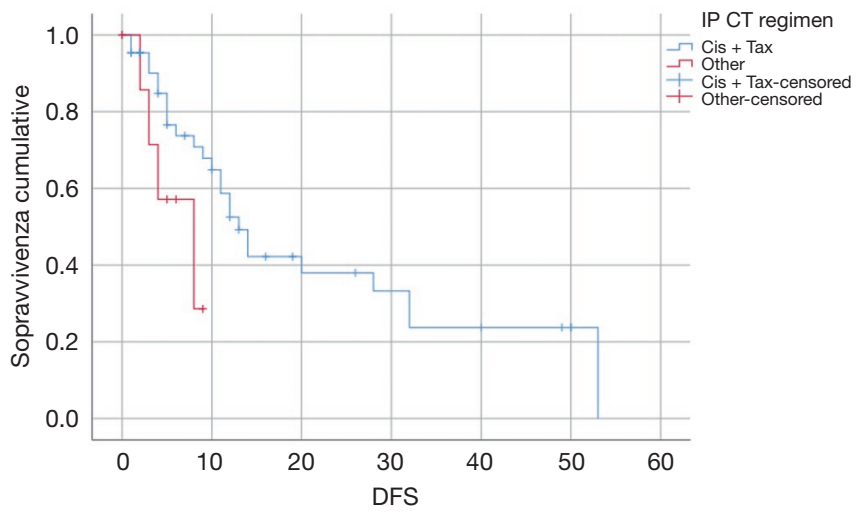

Figure 3 Kaplan-Meier survival curves of HIPEC cisplatin + paclitaxel vs. other HIPEC regimens.

was the only significant factor improving OS at multivariate analysis (OR 6.54, 95\% CI: 1.24-34.47, $\mathrm{P}=0.027$ ). Patients treated with IP Cisplatin + Taxol showed a median OS of 46 months (SD 6.4, 95\% CI: 33.4-58.6), while patients treated with other IP regimens showed a median OS of 12 months (SD 3.1, 95\% CI: $6.0-18.0$ ). The $2 \mathrm{y}-\mathrm{OS}$ was $72 \%$ and $3 \mathrm{y}-$ OS was $68 \%$ for Cisplatin + Taxol as IP CT, while the $2 y$ and $3 \mathrm{y}$-OS was $0 \%$ for other IP CT regimen (Figure 2).

Patients treated with IP Cisplatin + Taxol showed a median DFS of 13 months (SD 1.6, 95\% CI: 9.9-16.1), while patients treated with other IP regimens showed a median DFS of 8 months (SD 3.1, 95\% CI: 1.9-14.1) (Figure 3). Only tumor grading was the significant factor affecting DFS at univariate analysis (Table 12).

\section{Conclusion}

Intraperitoneal chemotherapy in ovarian cancer showed positive results that may be considered semi-definitive according to the level of evidence and should be maintained as a starting point for further investigations. At present intraperitoneal chemotherapy should be proposed to patients with advanced ovarian cancer as standard treatment at almost all disease stages. Platinum + taxane-based intraperitoneal regimens demonstrated superior results compared to other regimens. 


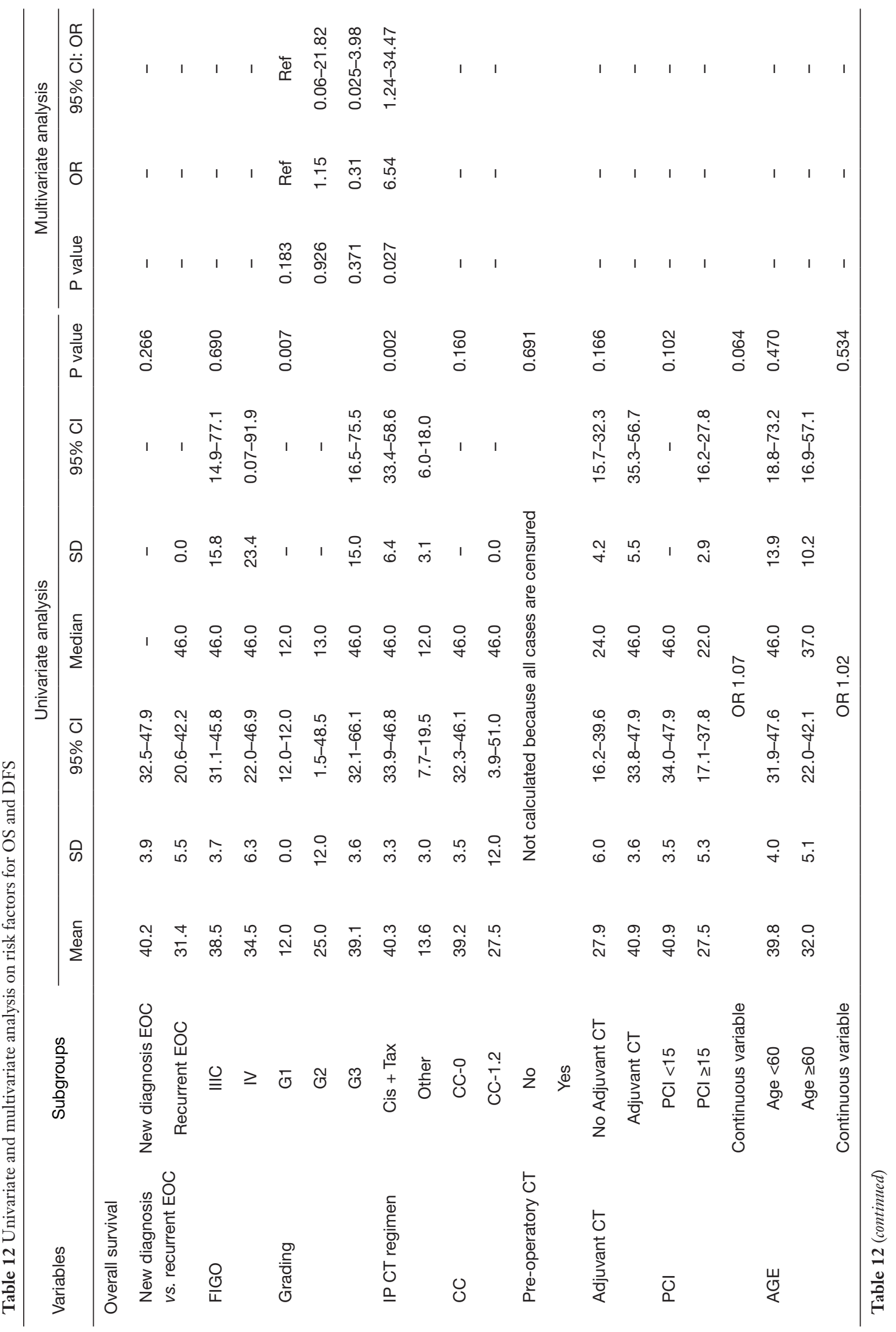




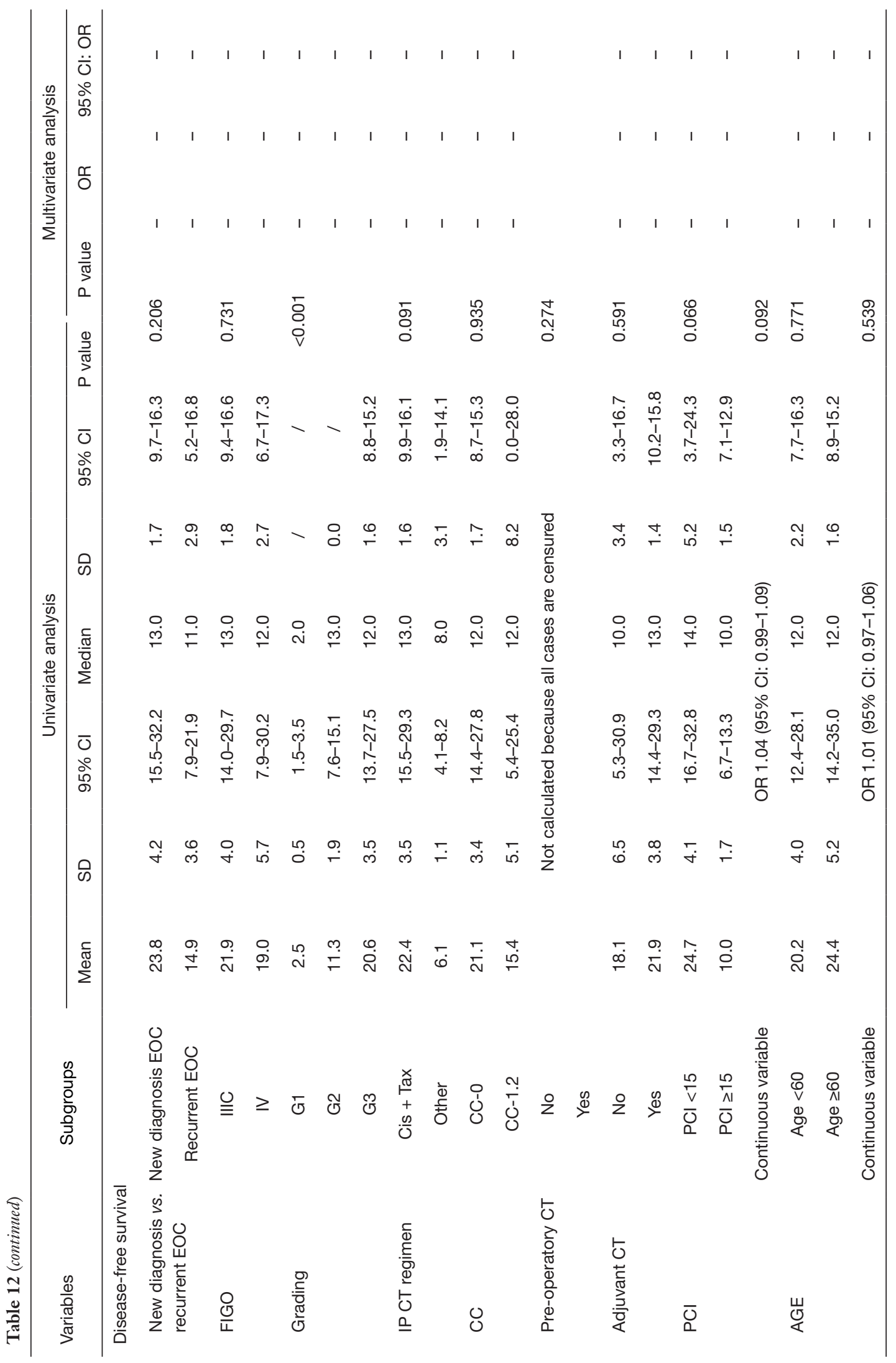




\section{Acknowledgments}

Funding: None.

\section{Footnote}

Provenance and Peer Review: This article was commissioned by the Guest Editors (Paul H. Sugarbaker and Kurt Van der Speeten) for the focused issue "Intraperitoneal Chemotherapy for Peritoneal Metastases: HIPEC, EPIC, NIPEC, PIPAC and More" published in Fournal of Gastrointestinal Oncology. This article has undergone external peer review.

Reporting Checklist: The authors have completed the PRISMA 2009 Checklist. Available at http://dx.doi. org/10.21037/jgo-2020-06

Conflicts of Interest: All authors have completed the ICMJE uniform disclosure form (available at http:// dx.doi.org/10.21037/jgo-2020-06). The focused issue was sponsored by the Peritoneal Surface Oncology Group International (PSOGI). The authors have no other conflicts of interest to declare.

Ethical Statement: The authors are accountable for all aspects of the work in ensuring that questions related to the accuracy or integrity of any part of the work are appropriately investigated and resolved.

Open Access Statement: This is an Open Access article distributed in accordance with the Creative Commons Attribution-NonCommercial-NoDerivs 4.0 International License (CC BY-NC-ND 4.0), which permits the noncommercial replication and distribution of the article with the strict proviso that no changes or edits are made and the original work is properly cited (including links to both the formal publication through the relevant DOI and the license). See: https://creativecommons.org/licenses/by-nc-nd/4.0/.

\section{References}

1. Prat J, FIGO Committee on Gynecologic Oncology. Staging classification for cancer of the ovary, fallopian tube, and peritoneum. Int J Gynaecol Obstet 2014;124:1-5.

2. Halkia E, Spiliotis J. The role of cytoreductive surgery and HIPEC in epithelial ovarian cancer. J BUON
2015;20:S12-28.

3. Riggs MJ, Pandalai PK, Kim J, et al. Hyperthermic Intraperitoneal Chemotherapy in Ovarian Cancer. Diagnostics (Basel, Switzerland) 2020;10:43.

4. Coleman RL, Monk BJ, Sood AK, et al. Latest research and clinical treatment of advanced-stage epithelial ovarian cancer. Nat Rev Clin Oncol 2013;10:211-24.

5. Ferrandina G, Legge F, Salutari V, et al. Impact of pattern of recurrence on clinical outcome of ovarian cancer patients: clinical considerations. Eur J Cancer 2006;42:2296-302.

6. Marchetti C, De Leo R, Musella A, D'Indinosante M, Capoluongo E, Minucci A, et al. BRCA Mutation Status to Personalize Management of Recurrent Ovarian Cancer: A Multicenter Study. Ann Surg Oncol 2018;25:3701-8.

7. Bristow RE, Tomacruz RS, Armstrong DK, et al. Survival effect of maximal cytoreductive surgery for advanced ovarian carcinoma during the platinum era: a metaanalysis. J Clin Oncol 2002;20:1248-59.

8. Vergote I, Harter P, Chiva L. Hyperthermic intraperitoneal chemotherapy does not improve survival in advanced ovarian cancer. Cancer 2019;125:4594-7.

9. Ushijima K. Treatment for recurrent ovarian cancer-at first relapse. J Oncol 2010;2010:497429.

10. Bakrin N, Classe JM, Pomel C, et al. Hyperthermic intraperitoneal chemotherapy (HIPEC) in ovarian cancer. J Visc Surg 2014;151:347-53.

11. Markman M, Francis P, Rowinsky E, et al. Intraperitoneal paclitaxel: a possible role in the management of ovarian cancer? Semin Oncol 1995;22:84-7.

12. Markman M. Intraperitoneal antineoplastic agents for tumors principally confined to the peritoneal cavity. Cancer Treat Rev 1986;13:219-42.

13. McClay EF, Howell SB. A review: intraperitoneal cisplatin in the management of patients with ovarian cancer. Gynecol Oncol 1990;36:1-6.

14. Cowan RA, O'Cearbhaill RE, Zivanovic O, et al. Current status and future prospects of hyperthermic intraoperative intraperitoneal chemotherapy (HIPEC) clinical trials in ovarian cancer. Int J Hyperthermia 2017;33:548-53.

15. Koole SN, Kieffer JM, Sikorska K, et al. Health-related quality of life after interval cytoreductive surgery with or without hyperthermic intraperitoneal chemotherapy (HIPEC) in patients with stage III ovarian cancer. Eur J Surg Oncol 2021;47:101-7.

16. van Driel WJ, Koole SN, Sonke GS. Hyperthermic Intraperitoneal Chemotherapy in Ovarian Cancer. N Engl J Med 2018;378:1363-4. 
17. Lim MC, Chang SJ, Yoo HJ, et al. Randomized trial of hyperthermic intraperitoneal chemotherapy (HIPEC) in women with primary advanced peritoneal, ovarian, and tubal cancer [abstract 5520]. J Clin Oncol 2017;35:5520.

18. Spiliotis J, Halkia E, Lianos E, et al. Cytoreductive Surgery and HIPEC in Recurrent Epithelial Ovarian Cancer: A Prospective Randomized Phase III Study. Ann Surg Oncol 2015;22:1570-5.

19. Chiva LM, Gonzalez-Martin A. A critical appraisal of hyperthermic intraperitoneal chemotherapy (HIPEC) in the treatment of advanced and recurrent ovarian cancer. Gynecol Oncol 2015;136:130-5.

20. Helm CW, Richard SD, Pan J, et al. Hyperthermic intraperitoneal chemotherapy in ovarian cancer first report of the HYPER-O registry. Int J Gynecol Cancer 2010;20:61-9.

21. Mikkelsen MS, Christiansen T, Petersen LK, et al. Morbidity after cytoreductive surgery and hyperthermic intraperitoneal chemotherapy with carboplatin used for ovarian, tubal, and primary peritoneal cancer. J Surg Oncol 2019;120:550-7.

22. Paris I, Cianci S, Vizzielli G, et al. Upfront HIPEC and bevacizumab-containing adjuvant chemotherapy in advanced epithelial ovarian cancer. Int J Hyperthermia 2018;35:370-4.

23. D'Hondt V, Goffin F, Roca L, et al. Interval Cytoreductive Surgery and Hyperthermic Intraperitoneal Chemotherapy in First-Line Treatment for Advanced Ovarian Carcinoma: A Feasibility Study. Int J Gynecol Cancer 2016;26:912-7.

24. Rettenmaier MA, Micha JP, Bohart R, et al.

A retrospective study comparing the efficacy of dosedense chemotherapy, intraperitoneal chemotherapy and dose-dense chemotherapy with hyperthermic intraperitoneal chemotherapy in the treatment of advanced stage ovarian carcinoma. Eur J Obstet Gynecol Reprod Biol 2020;244:101-5.

25. Biacchi D, Accarpio F, Ansaloni L, et al. Upfront debulking surgery versus interval debulking surgery for advanced tubo-ovarian high-grade serous carcinoma and diffuse peritoneal metastases treated with peritonectomy procedures plus HIPEC. J Surg Oncol 2019;120:1208-19.

26. Antonio CCP, Francisco-Cristobal MC, Alida GG, et al. Upfront cytoreduction and hyperthermic intraperitoneal chemotherapy with paclitaxel in patients with stage III-C serous epithelial ovarian cancer. Clin Exp Metastasis 2021;38:255.

27. Cascales-Campos P, López-López V, Gil J, et al. Hyperthermic intraperitoneal chemotherapy with paclitaxel or cisplatin in patients with stage III-C/IV ovarian cancer. Is there any difference? Surg Oncol 2016;25:164-70.

28. Ceresoli M, Verrengia A, Montori G, et al. Effect of cytoreductive surgery and hyperthermic intraperitoneal chemotherapy on relapse pattern in primary epithelial ovarian cancer: A propensity score based case-control study. J Gynecol Oncol 2018;29:e53.

29. Deraco M, Glehen O, Helm CW, et al. Cytoreductive Surgery \& Perioperative Chemotherapy for Peritoneal Surface Malignancy. Cinè-Med Publishing, Woodbury CT, USA, 2013.

30. Stathopoulos GP, Papadimitriou C, Aravantinos G, et al. Maintenance chemotherapy or not in ovarian cancer stages IIIA, B, C, and IV after disease recurrence. J BUON 2012;17:735-9.

31. Ledermann J, Harter P, Gourley C, et al. Olaparib maintenance therapy in patients with platinum-sensitive relapsed serous ovarian cancer: A preplanned retrospective analysis of outcomes by BRCA status in a randomised phase 2 trial. Lancet Oncol 2014;15:852-61.

32. Du Bois A, Vergote I, Gwenael F, et al. Randomized controlled phase III study evaluating the impact of secondary cytoreductive surgery in recurrent ovarian cancer: AGO DESKTOP III/ENGOT ov20. J Clin Oncol 2017;35:5501.

33. Amira G, Morsi A, Fayek IS, et al. Hyperthermic intraperitoneal chemotherapy versus systemic chemotherapy in recurrent platinum-sensitive ovarian cancer NCI case control study. Asian Pac J Cancer Prev 2019;20:621-7.

34. Arjona-Sanchez A, Rufian-Peña S, Artiles M, et al. Residual tumour less than 0.25 centimetres and positive lymph nodes are risk factors for early relapse in recurrent ovarian peritoneal carcinomatosis treated with cytoreductive surgery, HIPEC and systemic chemotherapy. Int J Hyperthermia 2018;34:570-7.

35. Baiocchi G, Ferreire F, Mantoan H, et al. Hyperthermic itraperitoneal Chemotherapy after Secondary Cytoreduction in Epithelial Ovarian Cancer: A SingleCenter Comparative Analysis. Ann Surg Oncol 2016;23:1294-301.

36. Bakrin N, Cotte E, Golfier F, et al. Cytoreductive surgery and hyperthermic intraperitoneal chemotherapy (HIPEC) for persistent and recurrent advanced ovarian carcinoma: A multicenter, prospective study of 246 patients. Ann Surg Oncol 2012;19:4052-8.

37. Carrabin N, Mithieux F, Meeus P, et al. Hyperthermic intraperitoneal chemotherapy with oxaliplatin and without 
adjuvant chemotherapy in stage IIIC ovarian cancer. Bull Cancer 2010;97:E23-32.

38. Cascales-Campos PA, Gil J, Feliciangeli E, et al. The Role of Hyperthermic Intraperitoneal Chemotherapy Using Paclitaxel in Platinum-Sensitive Recurrent Epithelial Ovarian Cancer Patients with Microscopic Residual Disease after Cytoreduction. Ann Surg Oncol 2015;22:987-93.

39. Chatzigeorgiou K, Economou S, Chrysafis G, et al. Treatment of Recurrent Epithelial Ovarian cancer with Secondart Cytoreduction and Continuous Intraoperative Intraperitoneal Hyperthermic Chemoperfusion (HIPEC). Zentralbl Gynakol 2003;125:424-9.

40. Cianci S, Ronsini C, Vizzielli G, et al. Cytoreductive surgery followed by HIPEC repetition for secondary ovarian cancer recurrence. Updates Surg 2019;71:389-94.

41. Classe JM, Glehen O, Decullier E, et al. Cytoreductive Surgery and Hyperthermic Intraperitoneal Chemotherapy for First Relapse of Ovarian Cancertle. Anticancer Res 2015;35:4997-5005.

42. Cotte E, Glehen O, Mohamed F, et al. Cytoreductive surgery and intraperitoneal chemohyperthermia for chemoresistant and recurrent advanced epithelial ovarian cancer: Prospective study of 81 patients. World J Surg 2007;31:1813-20.

43. Delotte J, Arias T, Guerin O, et al. Hyperthermic intraperitoneal chemotherapy for the treatment of recurrent ovarian cancer in elderly women. Acta Obstet Gynecol Scand 2015;94:435-9.

44. Deraco M, Rossi CR, Pennacchioli E, et al. Cytoreductive surgery followed by intraperitoneal hyperthermic perfusion in the treatment of recurrent epithelial ovarian cancer: A phase II clinical study. Tumori 2001;87:120-6.

45. Deraco $M$, Virzì $S$, Iusco DR, et al. Secondary cytoreductive surgery and hyperthermic intraperitoneal chemotherapy for recurrent epithelial ovarian cancer: A multi-institutional study. BJOG 2012;119:800-9.

46. Fagotti A, Paris I, Grimolizzi F, et al. Secondary cytoreduction plus oxaliplatin-based HIPEC in platinumsensitive recurrent ovarian cancer patients: A pilot study. Gynecol Oncol 2009;113:335-40.

47. Fagotti A, Costantini B, Petrillo M, et al. Cytoreductive surgery plus HIPEC in platinum-sensitive recurrent ovarian cancer patients: A case-control study on survival in patients with two year follow-up. Gynecol Oncol 2012;127:502-5.

48. Fagotti A, Petrillo M, Costantini B, et al. Minimally invasive secondary cytoreduction plus HIPEC for recurrent ovarian cancer: A case series. Gynecol Oncol 2014;132:303-6.

49. Fahim MI, Nassar OA, Mansour OM, et al. Combined cytoreductive surgery and hyperthermic intraperitoneal chemotherapy as a treatment for recurrent epithelial ovarian cancer-National Cancer Institute experience. J Egypt Natl Canc Inst 2018;30:139-41.

50. Furet E, Chéreau E, Lambaudie E, et al. Faisabilité, morbidité et survie de la chirurgie avec CHIP dans la prise en charge des récidives du cancer de l'ovaire. Gynecol Obstet Fertil 2013;41:493-8.

51. Gómez-Ruiz ÁJ, González-Gil A, Gil J, et al. Peritoneal Surface Disease Severity Score (PSDSS), AGO-score and TIAN model in patients with platinum-sensitive recurrent ovarian cancer treated by cytoreductive surgery plus HIPEC. Clin Exp Metastasis 2019;36:433-9.

52. Helm CW, Randall-Whitis L, Martin RS, et al. Hyperthermic intraperitoneal chemotherapy in conjunction with surgery for the treatment of recurrent ovarian carcinoma. Gynecol Oncol 2007;105:90-6.

53. Königsrainer I, Beckert S, Becker S, et al. Cytoreductive surgery and HIPEC in peritoneal recurrent ovarian cancer: Experience and lessons learned. Langenbecks Arch Surg 2011;396:1077-81.

54. Königsrainer I, Horvath P, Struller F, et al. Cytoreductive surgery and hyperthermic intraperitoneal chemotherapy in recurrent epithelial ovarian cancer with peritoneal metastases: A single centre experience. Langenbecks Arch Surg 2014;399:589-94.

55. Le Brun JF, Campion L, Berton-Rigaud D, et al. Survival Benefit of Hyperthermic Intraperitoneal Chemotherapy for Recurrent Ovarian Cancer: A Multi-institutional Case Control Study. Ann Surg Oncol 2014;21:3621-7.

56. Marocco F, Vaira M, Milani A, et al. Secondary cytoreductive surgery, hyperthermic intraperitoneal intraoperative chemotherapy, and chemotherapy alone: A retrospective comparison of alternative approaches in relapsed platinum sensitive ovarian cancer. Eur J Gynaecol Oncol 2016;37:638-43.

57. Muñoz-Casares FC, Rufián S, Rubio MJ, et al. The role of hyperthermic intraoperative intraperitoneal chemotherapy (HIPEC) in the treatment of peritoneal carcinomatosis in recurrent ovarian cancer. Clin Transl Oncol 2009;11:753-9.

58. Petrillo M, De Iaco P, Cianci S, et al. Long-Term Survival for Platinum-Sensitive Recurrent Ovarian Cancer Patients Treated with Secondary Cytoreductive Surgery Plus Hyperthermic Intraperitoneal Chemotherapy (HIPEC). 
Ann Surg Oncol 2016;23:1660-5.

59. Safra T, Grisaru D, Inbar M, et al. Cytoreduction surgery with hyperthermic intraperitoneal chemotherapy in recurrent ovarian cancer improves progression-free survival, especially in BRCA-positive patients-A casecontrol study. J Surg Oncol 2014;110:661-5.

60. Spiliotis JD, Iavazzo C, Kopanakis ND, et al. Secondary debulking for ovarian carcinoma relapse: The r-r dilemma - is the prognosis different for residual or recurrent disease? J Turk Ger Gynecol Assoc 2019;20:213-7.

61. van der Vange N, Van Goethem AR, Zoetmulder FAN, et al. Extensive cytoreductive surgery combined with intraoperative intraperitoneal perfusion with cisplatin under hyperthermic conditions (OVHIPEC) in patients with recurrent ovarian cancer: A feasibility pilot. Eur J Surg Oncol 2000;26:663-8.

62. Vernaccini N, Stefano B, Bertozzi S, et al. Surgical cytoreduction and hyperthermic intraperitoneal chemotherapy in patients affected by recurrent or persistent peritoneal carcinomatosis form epithelial ovarian cancer with high peritoneal cancer index values: Our single center experience. Eur J Surg Oncol 2016;42:S215.

63. Zivanovic O, Abramian A, Kullmann M, et al. HIPEC ROC I: A phase i study of cisplatin administered as hyperthermic intraoperative intraperitoneal chemoperfusion followed by postoperative intravenous platinum-based chemotherapy in patients with platinumsensitive recurrent epithelial ovarian cancer. Int J Cancer 2015;136:699-708.

64. Lu CH, Chang YH, Lee WH, et al. Second-Line Intraperitoneal Chemotherapy for Recurrent Epithelial Ovarian, Tubal and Peritoneal Cancer: A Propensity Score-Matching Study. Chemotherapy 2016;61:240-8.

65. Plaisant N, Quenet F, Fabbro M, et al. Secondary debulking surgery and intraperitoneal chemotherapy in advanced or recurrent epithelial ovarian cancer. Gynecol Obstet Fertil 2004;32:391-7.

66. Argenta PA, Sueblinvong T, Geller MA, et al. Hyperthermic intraperitoneal chemotherapy with carboplatin for optimally-cytoreduced, recurrent, platinum-sensitive ovarian carcinoma: A pilot study. Gynecol Oncol 2013;129:81-5.

67. Fagotti A, Costantini B, Vizzielli G, et al. HIPEC in recurrent ovarian cancer patients: Morbidity-related treatment and long-term analysis of clinical outcome. Gynecol Oncol 2011;122:221-5.

68. Petrillo M, Zucchettii M, Cianci S, et al. Pharmacokinetics of cisplatin during open and minimally-invasive secondary cytoreductive surgery plus HIPEC in women with platinum-sensitive recurrent ovarian cancer: A prospective study. J Gynecol Oncol 2019;30:e59.

69. Zanon C, Clara R, Chiappino I, et al. Cytoreductive surgery and intraperitoneal chemohyperthermia for recurrent peritoneal carcinomatosis from ovarian cancer. World J Surg 2004;28:1040-5.

70. Jandial DA, Brady WE, Howell SB,et al. A phase I pharmacokinetic study of intraperitoneal bortezomib andcarboplatin in patients with persistent or recurrent ovarian cancer: An NRGOncology/Gynecologic Oncology Group study. Gynecol Oncol 2017;145:236-42.

71. Tempfer CB, Celik I, Solass W, et al. Activity of Pressurized Intraperitoneal Aerosol Chemotherapy (PIPAC) with cisplatin and doxorubicin in women with recurrent, platinum-resistant ovarian cancer: Preliminary clinical experience. Gynecol Oncol 2014;132:307-11.

72. Tempfer CB, Winnekendonk G, Solass W, et al. Pressurized intraperitoneal aerosol chemotherapy in women with recurrent ovarian cancer: A phase 2 study. Gynecol Oncol 2015;137:223-8.

73. Tempfer CB, Giger-Pabst U, Seebacher V, et al. A phase I, single-arm, open-label, dose escalation study of intraperitoneal cisplatin and doxorubicin in patients with recurrent ovarian cancer and peritoneal carcinomatosis. Gynecol Oncol 2018;150:23-30.

74. Arjona-Sanchez A, Rufián-Peña S. Progress in the management of primary and recurrent ovarian carcinomatosis with peritonectomy procedure and HIPEC in a high volume centre. Int J Hyperthermia 2017;33:554-61.

75. Bakrin N, Bereder JM, Decullier E, et al. Peritoneal carcinomatosis treated with cytoreductive surgery and Hyperthermic Intraperitoneal Chemotherapy (HIPEC) for advanced ovarian carcinoma: A French multicentre retrospective cohort study of 566 patients. Eur J Surg Oncol 2013;39:1435-43.

76. Barakat RR, Sabbatini P, Bhaskaran D, et al. Intraperitoneal chemotherapy for ovarian carcinoma: Results of longterm follow-up. J Clin Oncol 2002;20:694-8.

77. Cascales Campos P, Gil J, Parrilla P. Morbidity and mortality outcomes of cytoreductive surgery and hyperthermic intraperitoneal chemotherapy in patients with primary and recurrent advanced ovarian cancer. Eur J Surg Oncol 2014;40:970-5.

78. Cripe J, Tseng J, Eskander R, et al. Cytoreductive Surgery and Hyperthermic Intraperitoneal Chemotherapy for Recurrent Ovarian Carcinoma: Analysis of 30-Day 
Morbidity and Mortality. Ann Surg Oncol 2015;22:655-61.

79. Di Giorgio A, Naticchioni E, Biacchi D, et al.

Cytoreductive surgery (peritonectomy procedures)

combined with hyperthermic intraperitoneal

chemotherapy (HIPEC) in the treatment of diffuse

peritoneal carcinomatosis from ovarian cancer. Cancer

2008;113:315-25.

80. Frenel JS, Leux C, Pouplin L, et al. Oxaliplatin-based hyperthermic intraperitoneal chemotherapy in primary or recurrent epithelial ovarian cancer: A pilot study of 31 patients. J Surg Oncol 2011;103:10-6.

81. Manzanedo I, Pereira F, Perez-Viejo E, et al.

Hyperthermic intraoperative intraperitoneal chemotherapy (HIPEC) with primary or secondary cytoreductive surgery in the treatment of adavanced epithelial ovarian cancer. Minerva Ginecol 2017;69:119-27.

82. Massari R, Barone M, Basilico R, et al. Peritonectomy and hyperthermic chemotherapy in patients with advanced or recurrent ephitelial ovarian cancer: a single center cohort study. Minerva Chir 2014;69:17-26.

83. Pavlov MJ, Kovacevic PA, Ceranic MS, et al. Cytoreductive surgery and modified heated intraoperative intraperitoneal chemotherapy (HIPEC) for advanced and recurrent ovarian cancer - 12-year single center experience. Eur J Surg Oncol 2009;35:1186-91.

84. Pavlov MJ, Ceranic MS, Latincic SM, et al. Cytoreductive surgery and hyperthermic intraperitoneal chemotherapy for the treatment of advanced epithelial and recurrent ovarian carcinoma: a single center experience. Int J Hyperthermia 2018;34:564-9.

85. Piso P, Dahlke MH, Loss M, et al. Cytoreductive surgery and hyperthermic intraperitoneal chemotherapy in peritoneal carcinomatosis from ovarian cancer. World J Surg Oncol 2004;2:21.

86. Raspagliesi F, Kusamura S, Campos Torres JC, et al. Cytoreduction combined with intraperitoneal hyperthermic perfusion chemotherapy in advanced/ recurrent ovarian cancer patients: The experience of National Cancer Institute of Milan. Eur J Surg Oncol 2006;32:671-5.

87. Robella M, Vaira M, Marsanic P, et al. Treatment of peritoneal carcinomatosis from ovarian cancer by surgical cytoreduction and hyperthermic intraperitoneal chemotherapy (HIPEC). Minerva Chir 2014;69:27-35.

88. Roviello F, Pinto E, Corso G, et al. Safety and potential benefit of hyperthermic intraperitoneal chemotherapy (HIPEC) in peritoneal carcinomatosis from primary or recurrent ovarian cancer. J Surg Oncol 2010;102:663-70.
89. Rufián S, Munoz-Casares FC, Briceno J, et al. Radical Surgery-Peritonectomy and Intraoperative Intraperitoneal Chemotherapy for the Treatment of Peritoneal Carcinomatosis in Recurrent or Primary Ovarian Cancer. J Surg Oncol 2006;94:316-24.

90. Sánchez-García S, Villarejo-Campos P, Padilla-Valverde D, et al. Intraperitoneal chemotherapy hyperthermia (HIPEC) for peritoneal carcinomatosis of ovarian cancer origin by fluid and $\mathrm{CO} 2$ recirculation using the closed abdomen technique (PRS-1.0 Combat): A clinical pilot study. Int J Hyperthermia 2016;32:488-95.

91. Sun JH, Sun JH, Yu Y, et al. Cytoreductive surgery plus hyperthermic intraperitoneal chemotherapy to treat advanced/recurrent epithelial ovarian cancer: Results from a retrospective study on prospectively established database. Transl Oncol 2016;9:130-8.

92. Warschkow R, Tarantino I, Lange J, et al. Does hyperthermic intraoperative chemotherapy lead to improved outcomes in patients with ovarian cancer? A single center cohort study in 111 consecutive patients. Patient Saf Surg 2012;6:12.

93. Yoshida Y, Sasaki H, Kurokawa T, et al. Efficacy of intraperitoneal continuous hyperthermic chemotherapy as consolidation therapy in patients with advanced epithelial ovarian cancer: A long-term follow-up. Oncol Rep 2005;13:121-5.

94. Ansaloni L, Agnoletti V, Amadori A, et al. Evaluation of extensive Cytoreductive Surgery and Hyperthermic Intraperitoneal Chemotherapy (HIPEC) in Patients with Advanced Epithelial Ovarian Cancer. Int J Gynecol Cancer 2012;22:778-85.

95. Ansaloni L, Coccolini F, Morosi L, et al. Pharmacokinetics of concomitant cisplatin and paclitaxel administered by hyperthermic intraperitoneal chemotherapy to patients with peritoneal carcinomatosis from epithelial ovarian cancer. Br J Cancer 2015;112:306-12.

96. Coccolini F, Campanati L, Catena F, et al. Hyperthermic intraperitoneal chemotherapy with cisplatin and paclitaxel in advanced ovarian cancer: A multicenter prospective observational study. J Gynecol Oncol 2015;26:54-61.

97. Gonzalez Bayon L, Steiner MA, Vasquez Jimenez W, et al. Cytoreductive surgery and hyperthermic intraperitoneal chemotherapy for the treatment of advanced epithelial ovarian carcinoma: Upfront therapy, at first recurrence, or later? Eur J Surg Oncol 2013;39:1109-15.

98. Pomel C, Ferron G, Lorimier G, et al. Hyperthermic intra-peritoneal chemotherapy using Oxaliplatin as consolidation therapy for advanced epithelial ovarian 
carcinoma. Results of a phase II prospective multicentre trial. CHIPOVAC study. Eur J Surg Oncol 2010;36:589-93.

99. Tentes AAK, Kakolyris S, Kyziridis D, et al. Cytoreductive surgery combined with hyperthermic intraperitoneal intraoperative chemotherapy in the treatment of advanced epithelial ovarian cancer. J Oncol 2012;2012:358341.

100.Harter P, Reuss A, Sehouli J, et al. Brief Report About the Role of Hyperthermic Intraperitoneal Chemotherapy in a Prospective Randomized Phase 3 Study in Recurrent Ovarian Cancer From Spiliotis et al. Int J Gynecol Cancer 2017;27:246-7.

101. Wang Y, Ren F, Chen P, et al. Effects of CytoReductive surgery plus hyperthermic IntraPEritoneal chemotherapy (HIPEC) versus CytoReductive surgery for ovarian cancer patients: A systematic review and meta-analysis. Eur J Surg Oncol 2019;45:301-9.

102.Zhang G, Zhu Y, Liu C, et al. The prognosis impact of hyperthermic intraperitoneal chemotherapy (HIPEC) plus cytoreductive surgery (CRS) in advanced ovarian cancer: The meta-analysis. J Ovarian Res 2019;12:33.

103.Piccart MJ, Floquet A, Scarfone G, et al. Intraperitoneal cisplatin versus no further treatment: 8-Year results of EORTC 55875, a randomized phase III study in ovarian cancer patients with a pathologically complete remission after platinum-based intravenous chemotherapy. Int J Gynecol Cancer 2003;13:196-203.

104. Tournigand C, Louvet C, Molitor JL, et al. Long-term survival with consolidation intraperitoneal chemotherapy for patients with advanced ovarian cancer with pathological complete remission. Gynecol Oncol 2003;91:341-5.

105. Gori J, Castano R, Toziano M, et al. Intraperitoneal Hyperthermic Chemotherapy in Ovarian cancer. Int J Gynecol Cancer 2005;15:233-9.

106. Kim JH, Lee JM, Ryu KS, et al. Consolidation hyperthermic intraperitoneal chemotherapy using paclitaxel in patients with epithelial ovarian cancer. J Surg Oncol 2010;101:149-55.

107. Mendivil AA, Rettenmaier MA, Abaid LN, et al. Consolidation hyperthermic intraperitoneal chemotherapy for the treatment of advanced stage ovarian carcinoma: a 3 year experience. Cancer Chemother Pharmacol 2017;80:405-10.

108. Rettenmaier MA, Mendivil AA, Abaid LN, et al. Consolidation hyperthermic intraperitoneal chemotherapy and maintenance chemotherapy following laparoscopic cytoreductive surgery in the treatment of ovarian carcinoma. Int J Hyperthermia 2015;31:8-14.

109. Suidan RS, St Clair CM, Lee SJ, et al. A comparison of primary intraperitoneal chemotherapy to consolidation intraperitoneal chemotherapy in optimally resected advanced ovarian cancer. Gynecol Oncol 2014;134:468-72.

110. Mousavi A, Karimi-Zarchi M, Behtash N, et al. The role of intraperitoneal carboplatin as consalidation chemotherapy in women with ovarian carcinoma: Report of our experience and systematic review. Int J Biomed Sci 2016;12:120-4.

111. Karadayi K, Yildiz C, Karakus S, et al. Cytoreductive surgery and perioperative intraperitoneal chemotherapy for gynecological malignancies: a single center experience. Eur J Gynaecol Oncol 2016;37:194-8.

112. Glehen O, Kwiatkowski F, Sugarbaker PH, et al. Cytoreductive surgery combined with perioperative intraperitoneal chemotherapy for the management of peritoneal carcinomatosis from colorectal cancer: a multiinstitutional study. J Clin Oncol 2004;22:3284-92.

113.McConnell YJ, Mack LA, Francis WP, et al. HIPEC + EPIC versus HIPEC-alone: differences in major complications following cytoreduction surgery for peritoneal malignancy. J Surg Oncol 2013;107:591-6.

114. Tan GHC, Ong WS, Chia CS, et al. Does early postoperative intraperitoneal chemotherapy (EPIC) for patients treated with cytoreductive surgery and hyperthermic intraperitoneal chemotherapy (HIPEC) make a difference? Int J Hyperthermia 2016;32:281-8.

115. Sugarbaker PH, Welch LS, Mohamed F, et al. A review of peritoneal mesothelioma at the Washington Cancer Institute. Surg Oncol Clin N Am 2003;12:605-21.

116. Van der Speeten K, Stuart OA, Sugarbaker PH. Pharmacology of perioperative intraperitoneal and intravenous chemotherapy in patients with peritoneal surface malignancy. Surg Oncol Clin N Am 2012;21:577-97.

117.Shi T, Jiang R, Pu H, et al. Survival benefits of dose-dense early postoperative intraperitoneal chemotherapy in frontline therapy for advanced ovarian cancer: a randomised controlled study. Br J Cancer 2019;121:425-8.

118. Los G, Mutsaers PH, van der Vijgh WJ, et al. Direct diffusion of cis-diamminedichloroplatinum(II) in intraperitoneal rat tumors after intraperitoneal chemotherapy: a comparison with systemic chemotherapy. Cancer Res 1989;49:3380-4.

119. Urano M, Kuroda M, Nishimura Y. For the clinical application of thermochemotherapy given at mild temperatures. Int J Hyperthermia 1999;15:79-107.

120. Conti M, De Giorgi U, Tazzari V, et al. Clinical Pharmacology of Intraperitoneal Cisplatin-Based 
Chemotherapy. J Chemother 2004;16:23-5.

121. Gladieff L, Chatelut E, Dalenc F, et al. Pharmacological bases of intraperitoneal chemotherapy. Bull Cancer 2009;96:1235-42.

122. Hakeam HA, Breakiet M, Azzam A, et al. The incidence of cisplatin nephrotoxicity post hyperthermic intraperitoneal chemotherapy (HIPEC) and cytoreductive surgery. Ren Fail 2014;36:1486-91.

123. Ceresoli M, Coccolini F, Ansaloni L. HIPEC and nephrotoxicity: A cisplatin induced effect? Eur J Surg Oncol 2016;42:909-10.

124. Rohena CC, Mooberry SL. Recent progress with microtubule stabilizers: new compounds, binding modes and cellular activities. Nat Prod Rep 2014;31:335-55.

125.Sugarbaker PH, Mora JT, Carmignani P, et al. Update on chemotherapeutic agents utilized for perioperative intraperitoneal chemotherapy. Oncologist 2005;10:112-22.

126. Mohamed F, Sugarbaker PH. Intraperitoneal taxanes. Surg Oncol Clin N Am 2003;12:825-33.

127.Lane P, Vichi P, Bain DL, Tritton TR. Temperature dependence studies of adriamycin uptake and cytotoxicity. Cancer Res 1987;47:4038-42.

128. Tritton TR. Cell surface actions of adriamycin. Pharmacol Ther 1991;49:293-309.

129. Van der Speeten K, Stuart OA, Mahteme H, et al. A pharmacologic analysis of intraoperative intracavitary cancer chemotherapy with doxorubicin. Cancer Chemother Pharmacol 2009;63:799-805.

130. Ozols RF, Locker GY, Doroshow JH, et al. Chemotherapy for murine ovarian cancer: a rationale for ip therapy with adriamycin. Cancer Treat Rep 1979;63:269-73.

131. Ozols RF, Locker GY, Doroshow JH, et al. Pharmacokinetics of adriamycin and tissue penetration in murine ovarian cancer. Cancer Res 1979;39:3209-14.

132. Ozols RF, Young RC, Speyer JL, et al. Phase I and pharmacological studies of adriamycin administered intraperitoneally to patients with ovarian cancer. Cancer Res 1982;42:4265-9.

133. Nagai K, Nogami S, Egusa H, et al. Pharmacokinetic evaluation of intraperitoneal doxorubicin in rats.

Cite this article as: Coccolini F, Fugazzola P, Montori G, Ansaloni L, Chiarugi M. Intraperitoneal chemotherapy for ovarian cancer with peritoneal metastases, systematic review of the literature and focused personal experience. J Gastrointest Oncol 2021;12(Suppl 1):S144-S181. doi: 10.21037/jgo-2020-06
Pharmazie 2014;69:125-7.

134.Pilati P, Mocellin S, Rossi CR, et al. Doxorubicin activity is enhanced by hyperthermia in a model of ex vivo vascular perfusion of human colon carcinoma. World J Surg 2003;27:640-6.

135. Salvatorelli E, De Tursi M, Menna P, et al. Pharmacokinetics of pegylated liposomal doxorubicin administered by intraoperative hyperthermic intraperitoneal chemotherapy to patients with advanced ovarian cancer and peritoneal carcinomatosis. Drug Metab Dispos 2012;40:2365-73.

136. Bachur NR, Gordon SL, Gee M V, et al. NADPH cytochrome $\mathrm{P}-450$ reductase activation of quinone anticancer agents to free radicals. Proc Natl Acad Sci U S A 1979;76:954-7.

137.Jacquet P, Averbach A, Stephens AD, et al. Heated intraoperative intraperitoneal mitomycin $\mathrm{C}$ and early postoperative intraperitoneal 5-fluorouracil: pharmacokinetic studies. Oncology 1998;55:130-8.

138. Piché N, Leblond FA, Sidéris L, et al. Rationale for heating oxaliplatin for the intraperitoneal treatment of peritoneal carcinomatosis: a study of the effect of heat on intraperitoneal oxaliplatin using a murine model. Ann Surg 2011;254:138-44.

139. De Somer F, Ceelen W, Delanghe J, et al. Severe hyponatremia, hyperglycemia, and hyperlactatemia are associated with intraoperative hyperthermic intraperitoneal chemoperfusion with oxaliplatin. Perit Dial Int 2008;28:61-6.

140. Cashin PH, Graf W, Nygren P, Mahteme H. Intraoperative hyperthermic versus postoperative normothermic intraperitoneal chemotherapy for colonic peritoneal carcinomatosis: a case-control study. Ann Oncol 2012;23:647-52.

141. Manzanedo I, Pereira F, Serrano Á, et al. The use of cisplatin plus doxorubicin or paclitaxel in hyperthermic intraperitoneal chemotherapy (HIPEC) for stage IIIC or IV epithelial ovarian cancer: a comparative study. Clin Transl Oncol 2019;21:1357-63. 